hep-ph/0109178

CERN-TH/2001-206

Edinburgh 2001-10

RM3-TH/01-08

\title{
Factorization and Resummation of Small $x$ Scaling Violations with Running Coupling
}

\author{
Guido Altarelli, ${ }^{(a)}$ Richard D. Ball ${ }^{(a, b)}$ and Stefano Forte ${ }^{(c), \dagger}$ \\ (a) Theory Division, CERN, \\ CH-1211 Genève 23, Switzerland. \\ (b) Department of Physics and Astronomy \\ University of Edinburgh, Edinburgh EH9 3JZ, Scotland \\ ${ }^{(c)}$ INFN, Sezione di Roma III \\ Via della Vasca Navale 84, I-00146 Rome, Italy
}

\begin{abstract}
We discuss the inclusion of running coupling effects in perturbative small $x$ evolution equations. We show that a running coupling BFKL-like $x$-evolution equation is fully compatible, up to higher twist corrections, with the standard factorized perturbative evolution of parton distributions. We then use this result, combined with the well-known Airy asymptotics, to prove that the oscillations which are present in the running-coupling BFKL solution do not affect the associated splitting functions, which instead remain smooth in the small $x$ limit. This allows us to give a prescription to include running-coupling corrections in the small- $x$ resummation of scaling violations. We show that these corrections are small in the HERA region.
\end{abstract}

CERN-TH/2001-206

September 2001

$\dagger$ On leave from INFN, Sezione di Torino, Italy 


\section{Introduction}

The theory of scaling violations of deep inelastic structure functions at small $x$ has recently attracted considerable interest, prompted by the extensive results obtained by experiments at HERA [1,2]. New effects beyond the low-order perturbative approximation [3-5] to anomalous dimensions or splitting functions should become important at small $x$. The BFKL approach [6-10] provides in principle a tool for the determination of the small $x$ improvements of the anomalous dimensions $[11,12]$. However, the data are in good agreement with a standard next-to-leading order perturbative treatment of scaling violations $[13,14,1,2]$, while naive attempts [15] to incorporate small $x$ logarithms fail completely $[16,17]$. Important progress has been made recently in understanding the empirical softness of the BFKL resummation [18-21]. Specifically, it is now understood that in order to avoid instabilities $[22,23]$, the inclusion of small $x$ contributions must be combined with a resummation of the collinear singularities of standard perturbative evolution.

An important aspect of the problem has to do with the implementation of running coupling effects. Indeed, it is a priori not obvious that the 'duality' relations $[11,24,19]$ which connect BFKL evolution in $\xi \equiv \ln \frac{1}{x}$ and renormalization-group evolution in $t \equiv \ln \frac{Q^{2}}{\mu^{2}}$ remain valid when the running of the coupling is taken into account. These duality relations play a crucial role $[19,20]$ in the desired simultaneous resummation of collinear singularities and small $x$ logs, and imply that in small- $x$ evolution factorization is preserved, namely, anomalous dimensions remain independent of the boundary conditions on the parton density.

In our previous work $[23,19,20]$ we have included running coupling effects perturbatively up to NLLx. We have shown that duality and factorization continue to hold, provided the anomalous dimension is supplemented by a term induced by the running of the coupling $[25,23]$. This term however corresponds to a singular contribution to the BFKL kernel, or equivalently, a perturbatively unstable contribution to the anomalous dimension. In $\overline{\mathrm{MS}}$ factorization this singular contribution is shifted to the quark sector [7]. Even though its effects are in practice rather small in the HERA region [20], this singularity signals a failure of the NLLx treatment of the running of the coupling in the asymptotic small $x$ limit.

In this paper, we address in detail the problems related to the inclusion of running coupling effects in small $x$ evolution equations. After reviewing the way duality is affected when the running of the coupling is included perturbatively, we consider the modified running-coupling BFKL $x$-evolution equation and its solution. We discuss the properties of the corresponding anomalous dimension and prove factorization and duality to all perturbative orders, up to higher twist corrections. ${ }^{1}$ We then study in detail the running coupling contribution to anomalous dimensions in the asymptotic small- $x$ limit, which may be determined exactly in terms of Airy functions, by taking a quadratic approximation of the BFKL kernel near its minimum [27]. In particular, we prove that the unphysical oscillatory behaviour which has been shown $[21,27-32]$ to characterize solutions to the runningcoupling BFKL equations at small $x$ does not affect the small $x$ asymptotics of splitting

1 Arguments for factorization have already been presented elsewhere [21,26], but they are partly based on model-dependent assumptions. 
functions. Therefore, the perturbative approach to scaling violations need not break down in the small $x$ limit. We then show that this quadratic approximation is sufficient to determine the leading $x \rightarrow 0$ asymptotics, since only the behaviour of the kernel near the minimum is relevant in this limit. Finally, we discuss how this asymptotic behaviour can be systematically matched to the small $x$ expansion of anomalous dimensions, and thus used to resum small $x$ running coupling terms. We show that the resummed result is free of unphysical singularities, perturbatively stable, and smooth in the small $x$ limit, and in the HERA region it only leads to a small correction to our previous phenomenological results.

\section{Perturbative Duality}

We start by reviewing the way duality of perturbative evolution is proven, first at fixed coupling and then perturbatively in the running coupling case. We then show how the perturbative expansion in the running coupling case is beset by unresummed singular contributions. The behaviour of structure functions at small $x$ is dominated by the large eigenvalue of evolution of the singlet parton component. Thus we consider the singlet parton density

$$
G(\xi, t)=x\left[g\left(x, Q^{2}\right)+k_{q} \otimes q\left(x, Q^{2}\right)\right],
$$

where $\xi=\log 1 / x, t=\log Q^{2} / \mu^{2}, g\left(x, Q^{2}\right)$ and $q\left(x, Q^{2}\right)$ are the gluon and singlet quark parton densities, respectively, and $k_{q}$ is such that, for each moment

$$
G(N, t)=\int_{0}^{\infty} d \xi e^{-N \xi} G(\xi, t)
$$

the associated anomalous dimension $\gamma\left(\alpha_{s}(t), N\right)$ corresponds to the largest eigenvalue in the singlet sector. The generalization to the full two-by-two matrix of anomalous dimensions is discussed in detail in ref. [20].

At large $t$ and fixed $\xi$ the evolution equation in $N$-moment space is then

$$
\frac{d}{d t} G(N, t)=\gamma\left(\alpha_{s}(t), N\right) G(N, t)
$$

where $\alpha_{s}(t)$ is the running coupling. The anomalous dimension is completely known at one- and two-loop level:

$$
\gamma\left(\alpha_{s}, N\right)=\alpha_{s} \gamma_{0}(N)+\alpha_{s}^{2} \gamma_{1}(N)+\ldots
$$

The corresponding splitting function is related by a Mellin transform to $\gamma\left(\alpha_{s}, N\right)$ :

$$
\gamma\left(\alpha_{s}, N\right)=\int_{0}^{1} d x x^{N} P\left(\alpha_{s}, x\right)
$$

Small $x$ for the splitting function corresponds to small $N$ for the anomalous dimension: more precisely $P \sim 1 / x(\log (1 / x))^{n}$ corresponds to $\gamma \sim n ! / N^{n+1}$. Even assuming that a 
leading twist description of scaling violations is still valid in some range of small $x$, as soon as $x$ is small enough that $\alpha_{s} \xi \sim 1$, with $\xi=\log 1 / x$, all terms of order $\left(\alpha_{s} / x\right)\left(\alpha_{s} \xi\right)^{n}$ (LLx) and $\alpha_{s}\left(\alpha_{s} / x\right)\left(\alpha_{s} \xi\right)^{n}$ (NLLx) which are present in the splitting functions must be considered in order to achieve an accuracy up to terms of order $\alpha_{s}^{2}\left(\alpha_{s} / x\right)\left(\alpha_{s} \xi\right)^{n}$ (NNLLx).

As is well known, these terms can be derived from the knowledge of the kernel

$$
\chi\left(\alpha_{s}, M\right)=\alpha_{s} \chi_{0}(M)+\alpha_{s}^{2} \chi_{1}(M)+\ldots .
$$

of the BFKL $\xi$-evolution equation

$$
\frac{d}{d \xi} G(\xi, M)=\chi\left(\alpha_{s}, M\right) G(\xi, M),
$$

which is satisfied at large $\xi$ by the inverse Mellin transform of the parton distribution

$$
G(\xi, M)=\int_{-\infty}^{\infty} d t e^{-M t} G(\xi, t) .
$$

This derivation was originally performed [11] at LLx by assuming the common validity of eq. (2.7) and eq. (2.3) in the region where $Q^{2}$ and $\xi$ are both large. However, it was more recently realized $[24,19,20]$ that the solution of eq. (2.7) coincides generally with that of eq. (2.3), up to higher twist corrections, provided only that the kernel of the former is related to that of the latter by a 'duality' relation, and boundary conditions are suitably matched. This implies that the domains of validity of these two equations are in fact the same in perturbation theory, up to power-suppressed corrections.

The derivation of duality is simplest when the coupling does not run, in which case the relation between the kernels of the two equations is

$$
\chi\left[\alpha_{s}, \gamma\left(\alpha_{s}, N\right)\right]=N .
$$

This result is immediately found by writing both eq. (2.3) and (2.7) and their solutions in terms of the inverse Mellin transform of the parton distribution

$$
G(N, M)=\int_{0}^{\infty} d \xi e^{-N \xi} G(\xi, M)=\int_{-\infty}^{\infty} d t e^{-M t} G(N, t) .
$$

The inverse Mellin of the solution to eq. (2.7) is

$$
G(N, M)=\frac{F_{0}(M)}{N-\chi\left(M, \alpha_{s}\right)},
$$

where $F_{0}(M)$ is an $N$-independent boundary condition. The large $t$ behaviour of $G(N, t)$ is determined by the rightmost singularity of $G(N, M)(2.11)$ in the $M$-plane, while the contributions of additional singularities further to the left are suppressed by powers of $Q^{2}$. In particular, perturbative singularities are given by solutions of the equation $\chi\left(\alpha_{s}, M\right)=$ $N$, while singularities of the boundary condition $F_{0}(M)$ are nonperturbative. 
Expanding the denominator of the solution (2.11) about its rightmost perturbative singularity, the solution eq. (2.11) is thus seen to coincide, up to higher twist and nonperturbative terms, with the inverse Mellin of the standard solution to the renormalization group eq. (2.3), namely

$$
G(N, M)=\frac{\tilde{F}_{0}(N)}{M-\gamma\left(N, \alpha_{s}\right)}
$$

provided the duality equation (2.9) holds, and the $t$-independent boundary condition $\tilde{F}_{0}(N)$ to eq. $(2.3)$ is related to $F_{0}(M)$ by

$$
\tilde{F}_{0}(N)=-\frac{F_{0}\left(\gamma\left(\alpha_{s}, N\right)\right)}{\chi^{\prime}\left(\gamma\left(\alpha_{s}, N\right)\right)} .
$$

This establishes the perturbative equivalence (duality) of eqs. (2.7) and (2.3), up to higher twist corrections. Using the perturbative expansion of $\chi(2.6)$ in the duality relation (2.9), we then find that $\chi_{0}$ determines $\gamma_{s}\left(\alpha_{s} / N\right)$, while $\alpha_{s} \chi_{1}$ leads to $\alpha_{s} \gamma_{s s}\left(\alpha_{s} / N\right)$, where the combination $\gamma_{s}\left(\alpha_{s} / N\right)+\alpha_{s} \gamma_{s s}\left(\alpha_{s} / N\right)$ correspond respectively to all terms of order $\left(\alpha_{s} / x\right)\left(\alpha_{s} \xi\right)^{n}$ and $\alpha_{s}\left(\alpha_{s} / x\right)\left(\alpha_{s} \xi\right)^{n}$ in the splitting functions.

When one goes beyond LLx, i.e. beyond the leading-order approximation for $\chi$, the running of the coupling cannot be neglected, and this derivation must be re-examined. Indeed, in $M$ space the usual running coupling $\alpha_{s}(t)$ becomes a differential operator: taking only the one-loop beta function into account

$$
\widehat{\alpha}_{s}=\frac{\alpha_{s}}{1-\beta_{0} \alpha_{s} \frac{d}{d M}}+\cdots
$$

where $\beta_{0}$ is the first coefficient of the $\beta$-function (so $\beta=-\beta_{0} \alpha_{s}^{2}+\cdots$ ), with the obvious generalization to higher orders. Hence, assuming the coupling to run in the usual way with $Q^{2}$, the $\xi$-evolution equation eq. (2.7) becomes [27]

$$
\frac{d}{d \xi} G(\xi, M)=\chi\left(\widehat{\alpha}_{s}, M\right) G(\xi, M)
$$

where the derivatives with respect to $M$ act on everything to the right, and $\chi$ may be expanded as in eq. (2.6) keeping the powers of $\widehat{\alpha}_{s}$ on the left. Clearly, an operator-valued evolution kernel is a potential threat to factorization. Furthermore, one may ask whether eq. (2.15) is indeed the proper form of the running-coupling BFKL equation. Here we will show that, besides being intuitively appealing, the evolution eq. (2.15) has the property that its solutions are still the same as those of a renormalization-group equation eq. (2.3), with a suitably matched kernel, to all perturbative orders and up to power corrections. This implies consistency of eq. (2.15) with standard perturbative evolution, and in particular that factorization is preserved. Therefore, eq. (2.15) can be viewed as an alternative representation of the standard renormalization-group equation.

It is clear from eq. (2.15) that running coupling effects begin at NLLx. In fact, to $\mathrm{N}^{k} \mathrm{LLx}$ it is sufficient to retain the first $k$ terms in the expansion of $\widehat{\alpha}_{s}(2.14)$ in powers of $\alpha_{s}$; the ensuing equation can then be solved perturbatively. To NLLx one finds [23] that 
this solution is again the same as that of a dual $t$-evolution equation (2.3), provided the fixed-coupling duality relation eq. (2.9) is modified by letting $\alpha_{s} \rightarrow \alpha_{s}(t)$, and then by adding to $\gamma_{s s}$ an extra term [25] $\Delta \gamma_{s s}$ proportional to $\beta_{0}$ :

$$
\Delta \gamma_{s s}\left(\frac{\alpha_{s}}{N}\right)=-\beta_{0} \frac{\chi_{0}^{\prime \prime}\left(\gamma_{s}\right) \chi_{0}\left(\gamma_{s}\right)}{2 \chi_{0}^{\prime 2}\left(\gamma_{s}\right)}
$$

Equivalently, the duality relation eq. (2.9) can be formally preserved, provided that $\alpha_{s} \rightarrow$ $\alpha_{s}(t)$ and the function $\chi$ used in it is no longer identified with the BFKL kernel, but rather given by an 'effective' $\chi$ function

$$
\begin{gathered}
\chi_{\mathrm{eff}}\left(\alpha_{s}, M\right)=\chi\left(\alpha_{s}, M\right)+\Delta \chi\left(\alpha_{s}, M\right), \\
\Delta \chi\left(\alpha_{s}, M\right)=\alpha_{s}^{2} \Delta \chi_{1}(M)+\ldots,
\end{gathered}
$$

where $\chi\left(\alpha_{s}, M\right)$ is obtained letting $\widehat{\alpha}_{s} \rightarrow \alpha_{s}$ in the kernel of eq. (2.15), ${ }^{2}$ and the correction term $\Delta \chi$ to NLLx is given by

$$
\Delta \chi_{1}(M)=\beta_{0} \frac{\chi_{0}^{\prime \prime}(M) \chi_{0}(M)}{2 \chi_{0}^{\prime}(M)}
$$

Also, the matching of boundary conditions is now given by a more complicated relation than eq. (2.13), involving derivatives of $F_{0}$ and $\chi_{0}$; however, it remains independent of $t$, and only involves $N$ and $\alpha_{s}$ (at the initial scale). We have further verified by explicit computation that if the perturbative solution of eq. (2.15) with the running coupling eq. (2.14) is pursued up to $\mathrm{N}^{2} \mathrm{LLx}$ these results remain valid: the boundary condition remains $t$-independent, and duality can be preserved, provided only a further $\Delta \chi_{2}$ term is included in $\chi_{\text {eff }}$ eq. $(2.17)$.

The problem with the perturbative approach is that the correction terms which must be included in the effective $\chi$ eq. (2.17) have an unphysical singularity: $\chi_{0}(M)$ has a minimum at $M=\frac{1}{2}$, so the denominator of $\Delta \chi_{1}(M)$ vanishes, resulting in a simple pole in the NLLx correction $\Delta \chi_{1}$ eq. (2.18) at $M=\frac{1}{2}$. The NNLLx correction $\Delta \chi_{2}$ turns out to have a fourth-order pole, and in fact at each extra order three extra powers of $\left(\chi_{0}^{\prime}\right)^{-1}$ appear. As explained in ref. [23], this leads to a perturbative instability: as a consequence of the singularity, the splitting function $\Delta P_{s s}$ eq. (2.5) associated with the anomalous dimension $\Delta \gamma_{s s}$ behaves as

$$
\frac{\Delta P_{s s}\left(\alpha_{s}, \xi\right)}{P_{s}\left(\alpha_{s}, \xi\right)} \underset{\xi \rightarrow \infty}{\sim}\left(\alpha_{s} \xi\right)^{2}
$$

In practice, the coefficient of this rise turns out to be small enough that its effects are negligible in the HERA region [20]. However, the situation remains unsatisfactory from a theoretical point of view: it would be better to extend the perturbative proof of factorization and duality to the case when the running of the coupling is included to all orders, and then sum up these perturbatively unstable terms. In the sequel, we will do this, and show that the $M=\frac{1}{2}$ singularity and the corresponding ones that appear at higher orders in $\alpha_{s} \beta_{0}$ are an artifact of the expansion, are not present in the all-order solution, and can thus be resummed.

2 Notice that, beyond leading order, this is not the same as the fixed-coupling kernel of eq. (2.7), which refers to the unphysical case of a theory where $\beta_{0}=0$ identically. 


\section{Running Coupling Duality and Factorization}

In this section, we prove to all orders the perturbative duality and factorization introduced in the previous section, and we discuss some general properties of the all-order solution to the running coupling BFKL equation, specifically its apparently unphysical behaviour in the $\xi \rightarrow 0$ limit. We start from the running coupling $\xi$ evolution equation (2.15). We consider first the case in which the running is included at one loop according to eq. (2.14), and the kernel $\chi$ is linear in the operator $\widehat{\alpha}_{s}$ :

$$
\chi\left(\alpha_{s}, M\right)=\widehat{\alpha}_{s} \varphi\left(\alpha_{s}, M\right),
$$

where $\varphi$ is a function of $M$ and the fixed coupling $\alpha_{s}$. This is of course the case when the leading order form of $\chi$ is considered, in which case $\varphi=\chi_{0}(M)$, but also more generally whenever terms of higher order in the fixed coupling $\alpha_{s}$ are included in $\varphi$, as in the case of the resummed leading order kernel $\tilde{\chi}_{0}$ previously discussed by us $[19,20]$. In the remainder of this section we will omit the explicit dependence of $\varphi$ on $\alpha_{s}$, since it will not play any role in the ensuing proof of factorization and duality. The generalization of the proof to higher orders in the running coupling $\alpha_{s}(t)$ will be discussed at the end of the section. In sect. 5 we will then show that, in order to perform a running coupling resummation to NLLx, it is sufficient to consider a kernel $\chi$ of the form of eq. (3.1), and we will also discuss higher order generalizations.

With the kernel (3.1), after taking a second Mellin transform (2.2), eq. (2.15) becomes the differential equation

$$
\left(1-\beta_{0} \alpha_{s} \frac{d}{d M}\right) N G(N, M)+F(M)=\alpha_{s} \varphi(M) G(N, M)
$$

The function $F(M)$ is the boundary condition, obtained by acting with the operator (1 $\left.\beta_{0} \alpha_{s} \frac{d}{d M}\right)$ on the boundary condition $-F_{0}(M)$ of eq. (2.12). The perturbative solution [23] to eq. (3.2) discussed in the previous section is an expansion in $\alpha_{s}$ at fixed $\alpha_{s} / N$ and it can be built up by an iterative procedure: at order $n$ the order $n-1$ expression for $G(N, M)$ is inserted in the right-hand side. Clearly, this iterative solution is a linear functional of $F(M)$.

However, it is also easy to determine the general solution to the differential equation $[27,28]$ :

$$
G(N, M)=H(N, M)+\int_{M_{0}}^{M} d M^{\prime} \frac{H(N, M)}{H\left(N, M^{\prime}\right)} \frac{F\left(M^{\prime}\right)}{\beta_{0} \alpha_{s} N},
$$

where $H(N, M)$ is the solution of the homogeneous equation

$$
H(N, M)=H\left(N, M_{0}\right) \exp \left[\frac{M-M_{0}}{\beta_{0} \alpha_{s}}-\frac{1}{\beta_{0} N} \int_{M_{0}}^{M} d M^{\prime} \varphi\left(M^{\prime}\right)\right]
$$

with an arbitrary initial condition $H\left(N, M_{0}\right)$. The inhomogeneous term can be written as

$$
G(N, M)=\int_{M_{0}}^{M} d M^{\prime} \exp \left[\frac{M-M^{\prime}}{\beta_{0} \alpha_{s}}-\frac{1}{\beta_{0} N} \int_{M^{\prime}}^{M} d M^{\prime \prime} \varphi\left(M^{\prime \prime}\right)\right] \frac{F\left(M^{\prime}\right)}{\beta_{0} \alpha_{s} N},
$$


and thus depends linearly on $F(M)$ while being independent of $H\left(N, M_{0}\right)$.

Let us now see how the iterative solution can be recovered from the general solution, by expanding it in $\alpha_{s}$ at fixed $\alpha_{s} / N$ : the iterative solution corresponds to the inhomogeneous term in eq. (3.5), while the homogeneous solution in eq. (3.4) vanishes faster than any fixed perturbative order in $\beta_{0}$. To see how this works, change the integration variable in eq. (3.3) from $M^{\prime}$ to

$$
y\left(M, M^{\prime}\right)=-\frac{1}{\beta_{0} \alpha_{s} N} \int_{M^{\prime}}^{M}\left[N-\alpha_{s} \varphi\left(M^{\prime \prime}\right)\right] d M^{\prime \prime}
$$

The inhomogeneous solution (3.5) then becomes

$$
G(N, M)=\int_{0}^{y\left(M, M_{0}\right)} e^{-y} \frac{F\left(M^{\prime}(y, M)\right)}{\left[N-\alpha_{s} \varphi\left(M^{\prime}(y, M)\right)\right]} d y
$$

where $M^{\prime}$ is implicitly defined as a function $M^{\prime}=M^{\prime}(y, M)$ by eq. (3.6). The expansion of $M^{\prime}(y, M)$ in powers of $\alpha_{s}$ is

$$
M^{\prime}=M+\frac{\beta_{0} \alpha_{s} N}{\left[N-\alpha_{s} \varphi(M)\right]} y+O\left(\alpha_{s}^{2}\right)
$$

Using this expansion to do the integral, and noting that $y \rightarrow \infty$ in the limit $\alpha_{s} \rightarrow 0$, one finds

$$
G(N, M)=\frac{F(M)}{\left[N-\alpha_{s} \varphi(M)\right]}+\frac{\alpha_{s} \beta_{0}}{\left[N-\alpha_{s} \varphi(M)\right]} \frac{d}{d M} \frac{F(M)}{\left[N-\alpha_{s} \varphi(M)\right]}+\ldots,
$$

which is the iterative solution of ref. [23], discussed in the previous section. Furthermore, the homogeneous solution (3.4) is manifestly proportional to $e^{-y}$ and therefore vanishes to all orders in the perturbative expansion. Thus in the following we drop the homogeneous term and study only the inhomogeneous term (3.5), which reproduces the perturbative solution.

Starting from $G(N, M)$ one can do either of the inverse Mellin transforms, and obtain $G(\xi, M)$ or $G(N, t)$. We consider $G(N, t)$ first, in order to prove that it satisfies a renormalization-group equation of the usual form, and study the modification induced by running-coupling effects on the anomalous dimension and the duality equation which relates it to the kernel $\chi$. By taking the inverse $M$-Mellin transform we can write $G(N, t)$ in the form

$$
G(N, t)=\int_{c-i \infty}^{c+i \infty} \frac{d M}{2 \pi i} \exp \left[M t+\frac{M-M_{0}}{\beta_{0} \alpha_{s}}-\frac{1}{\beta_{0} N} \int_{M_{0}}^{M} d M^{\prime \prime} \varphi\left(M^{\prime \prime}\right)\right] \frac{I(N, M)}{N}
$$

where

$$
I(N, M) \equiv \int_{M_{0}}^{M} d M^{\prime} \exp \left[\frac{M_{0}-M^{\prime}}{\beta_{0} \alpha_{s}}+\frac{1}{\beta_{0} N} \int_{M_{0}}^{M^{\prime}} d M^{\prime \prime} \varphi\left(M^{\prime \prime}\right)\right] \frac{F\left(M^{\prime}\right)}{\beta_{0} \alpha_{s}}
$$


It is well-known $[28,25]$ that evaluating the $M$ integral by saddle point one recovers a running-coupling version of the duality relation eq. (2.9). However, both duality and perturbative factorization seem to be spoiled in the process. We now review this derivation, and then prove that, contrary to appearances, factorization still holds in the perturbative limit, and duality is recovered with a series of corrections eq. (2.17).

The saddle-point condition for the integrand of eq. (3.10) (assuming $I(N, M)$ to be a smooth function of $M$ ) is

$$
t+\frac{1}{\beta_{0} \alpha_{s}}-\frac{1}{\beta_{0} N} \varphi\left(M_{s}\right)=0
$$

Noting that at leading order $t+1 /\left(\beta_{0} \alpha_{s}\right)=\left[\beta_{0} \alpha_{s}(t)\right]^{-1}$, we see that this is equivalent to

$$
\alpha_{s}(t) \varphi\left(M_{s}\right)=N
$$

Identifying $M_{s}$ with the anomalous dimension,

$$
M_{s}=\gamma_{s}\left(\alpha_{s}(t) / N\right),
$$

we see that eq. (3.13) coincides with the duality relation, eq. (2.9), but with the fixed coupling replaced by the running coupling $[28,25]$. Substituting back the saddle condition in the exponent of eq. (3.10) we get (differentiating the duality relation with respect to $t$ )

$$
\frac{M_{s}}{\alpha_{s}(t) \beta_{0}}-\frac{M_{0}}{\alpha_{s} \beta_{0}}-\frac{1}{\beta_{0} \alpha_{s} N} \int_{M_{0}}^{M_{s}} d M^{\prime} \varphi\left(M^{\prime}\right)=\int_{t_{0}}^{t} d t^{\prime} \gamma_{s}\left(\alpha_{s}\left(t^{\prime}\right) / N\right),
$$

where $\gamma_{s}\left(\alpha_{s}\left(t^{\prime}\right) / N\right)$ is defined for all $\alpha_{s}\left(t^{\prime}\right)$ as the solution of eq. (3.14), and we choose for simplicity (and with no loss of generality) $\alpha_{s}$ such that $\alpha\left(t_{0}\right)=\alpha_{s}$. Performing the saddle integral we thus find

$$
G(N, t)=\sqrt{\frac{N \beta_{0} \alpha_{s}}{-\varphi^{\prime}\left[M_{s}(t)\right]}} \exp \left(\int_{t_{0}}^{t} d t^{\prime} \gamma_{s}\left(\alpha\left(t^{\prime}\right), N\right)\right) \frac{I\left(N, M_{s}(t)\right)}{N}+\cdots,
$$

where the dots denote corrections to the leading saddle-point approximation.

Eq. (3.16) seems to violate factorization because of the $t$-dependence induced by the substitution of the saddle condition $M=M_{s}(t)$ in the integral $I(N, M)$, which depends on the boundary condition. Also, it seems to violate duality because the $t$-dependent prefactor $1 / \sqrt{\varphi^{\prime}}$ spoils the identification of the anomalous dimensions with $\gamma_{s}$. This is puzzling because these violations seem to start at NLLx, whereas the explicit perturbative calculation discussed above shows that factorization and duality are respected at NLLx. We now address both issues in turn.

First, let us consider the integral $I\left(N, M_{s}(t)\right)$ which appears in eq. (3.16) (with $I(N, M)$ defined in eq. (3.11) and $M_{s}$ eq. (3.14)) in the perturbative LLx and leading-log $Q^{2} \operatorname{limit}$, i.e. as $\alpha_{s} \rightarrow 0$, with $\alpha_{s} / N$ and $\alpha_{s} t$ fixed. We change the integration variable from $M^{\prime}$ to $y\left(M_{0}, M^{\prime}\right)$ defined in eq. (3.6), so we can write this integral in the form

$$
I\left(N, M_{s}(t)\right)=\int_{0}^{y[\gamma(\alpha(t) / N)]} d y e^{-y} \frac{F\left(M^{\prime}\left(y, M_{0}\right)\right)}{\left[N-\varphi\left(M^{\prime}\left(y, M_{0}\right)\right)\right]}
$$


We take $\left(1-\frac{\alpha_{s}}{N} \varphi\right)<0$ and $M^{\prime}<M_{0}$, so that $y>0$. As a result, in the perturbative limit, $y(\gamma) \rightarrow \infty$ and the perturbative $t$ dependence disappears to all orders in the expansion. So perturbatively $I\left(N, M_{s}(t)\right)$ is independent of $t$ to all orders, even though it does depend on $t_{0}$ because $M^{\prime}=M^{\prime}\left(y, M_{0}\right)$. This proves factorization to all perturbative orders: the $t$ dependence of $G(N, t)$ is entirely determined by the evolution kernel $\chi$, and does not depend on the boundary condition

The $t$-dependence, however, is not only due to the anomalous dimensions $\gamma_{s}\left(\alpha_{s}(t) / N\right)$ which satisfies the running-coupling duality relation eqs. (3.13),(3.14), but also to the square-root prefactor in eq. (2.16). However, because this additional $t$-dependence goes entirely through the running coupling $\alpha_{s}(t)$, it has the structure of a NLLx coefficient function, and it can thus be reabsorbed by small $-x$ scheme change $[23,20,33]$ in a redefinition of the anomalous dimension and the boundary condition:

$$
\begin{aligned}
\frac{1}{\sqrt{-\varphi^{\prime}\left(\gamma_{s}\left(\frac{\alpha_{s}(t)}{N}\right)\right)}} & =\exp -\left[\frac{1}{2} \ln -\varphi^{\prime}\left(\gamma_{s}\left(\frac{\alpha_{s}(t)}{N}\right)\right)\right] \\
& =\exp \left[\beta_{0} \int_{t_{0}}^{t} d t^{\prime} \frac{\varphi^{\prime \prime}\left(\frac{\alpha_{s}\left(t^{\prime}\right)}{N}\right) \varphi\left(\frac{\alpha_{s}\left(t^{\prime}\right)}{N}\right)}{2 \varphi^{\prime 2}\left(\frac{\alpha_{s}\left(t^{\prime}\right)}{N}\right)} \alpha_{s}\left(t^{\prime}\right)\right] \frac{1}{\sqrt{-\varphi^{\prime}\left(\gamma_{s}\left(\frac{\alpha_{s}\left(t_{0}\right)}{N}\right)\right)}} \\
& =\exp \left[\int_{t_{0}}^{t} d t^{\prime} \Delta \gamma_{s s}\left(\alpha_{s}\left(t^{\prime}\right) / N\right)\right] \frac{1}{\sqrt{-\varphi^{\prime}\left(\gamma_{s}\left(\frac{\alpha_{s}\left(t_{0}\right)}{N}\right)\right)}}
\end{aligned}
$$

where the second equality follows from differentiating the duality relation, and the last factor, which depends on the initial scale $t_{0}$ only, can be reabsorbed in the boundary condition. Identifying $\varphi=\chi_{0}$ we see immediately that the resulting addition to $\gamma, \Delta \gamma_{s s}$, is the same as that determined perturbatively at NLLx eq. (2.16).

Furthermore, we can use the saddle-point method to generate an asymptotic expansion of $G(N, t)$. Noting that the first correction to the gaussian approximation is a cubic term in $M-M_{s}$ proportional to $\varphi^{\prime}$, and that odd terms in the expansion vanish upon integration, this is seen to generate an expansion in powers of

$$
\frac{N \beta_{0}}{\varphi^{\prime 3}}=\alpha_{s}\left(\frac{\alpha_{s}^{2}}{N^{2}}\right)^{2}\left[\gamma_{s}^{\prime}\left(\alpha_{s}(t) / N\right)\right]^{3} \beta_{0},
$$

where $\gamma_{s}^{\prime}$ is differentiated with respect to its argument $\alpha_{s}(t) / N$. It follows that higher orders in the expansion give a series of terms which can be absorbed in a series of contributions to the anomalous dimension of the form

$$
\gamma_{\mathrm{rc}}\left(\alpha_{s}(t), N\right)=\gamma_{s}\left(\frac{\alpha_{s}(t)}{N}\right)+\beta_{0} \alpha_{s}(t) \Delta \gamma_{s s}\left(\frac{\alpha_{s}(t)}{N}\right)+\left[\beta_{0} \alpha_{s}(t)\right]^{2} \Delta \gamma_{s s s}\left(\frac{\alpha_{s}(t)}{N}\right)+\ldots
$$

where $\Delta \gamma_{s s}$, given by eq. (2.16), is due to the leading fluctuations about the saddle, $\Delta \gamma_{s s s}$ comes from the next-to-leading fluctuations and so on. It is also easy to check that the boundary condition does not contribute to this additional $t$ dependence, because all its higher order derivatives with respect to $M$ vanish in the perturbative limit, for the same reason that the homogeneous term vanishes to all perturbative orders. 
This shows that we can formally preserve the duality relation eq. (2.9), with $\alpha_{s} \rightarrow$ $\alpha_{s}(t)$ and an effective $\chi_{\text {eff }}$ eq. (2.17), provided at each order in $\alpha_{s}$ we add a new correction term to the kernel $\chi$, determined from $\gamma_{\mathrm{rc}}$ eq. (3.20); the first correction is given by eq. (2.18). The order $\alpha_{s}^{k}$ correction is proportional to $\left[\varphi^{\prime}\right]^{-3 k}$ eq. (3.19), and thus if $\varphi$ has a minimum, the correction has a $3 k$-th order pole as $M \rightarrow M_{\min }$. Therefore [23], when $\xi \rightarrow \infty$ the corresponding splitting function rises by a factor $\xi^{2 k}$ faster than the leading order, and the perturbative expansion is unstable. These singular contributions will be resummed in sect. 4-5, leading to an anomalous dimension $\gamma$ and associated kernel $\chi_{\text {eff }}$ which are free of singularities, and thus have stable perturbative expansions.

Before we turn to this task, let us exploit further the factorization property which follows from the fact that the integral $I\left(N, M_{s}(t)\right)$ in eq. (3.17) is asymptotically a function of $N$, independent of $t$ to all orders in the perturbative expansion in $\alpha_{s}(t)$. This implies that, in the same limit, $G(N, t)$ in eq. (3.16) can be written as

$$
\begin{aligned}
G(N, t)= & \int_{c-i \infty}^{c+i \infty} \frac{d M}{2 \pi i} e^{M t} \\
& \quad \exp \left[\frac{M-M_{0}}{\beta_{0} \alpha_{s}}-\frac{1}{\beta_{0} N} \int_{M_{0}}^{M} d M^{\prime \prime} \varphi\left(M^{\prime \prime}\right)\right] \frac{I\left(N, t_{0}\right)}{N} .
\end{aligned}
$$

Note that this is the same form that one could obtain from $G(N, M)$ given by the homogeneous solution in eq. (3.4) with the identification $H\left(N, M_{0}\right)=I\left(N, t_{0}\right) / N$, that is with an initial condition which is a linear functional of $F(M)$.

This proof of factorization and duality can be extended to the case in which the running of the coupling is included to higher orders. To this purpose, introduce a perturbative expansion of the inverse $M$-Mellin $G(\xi, t)$ of the solution to eq. (2.15) in powers of $\alpha_{s}(t)$ :

$$
G(\xi, t)=G_{0}(\xi, t)+\alpha_{s}(t) G_{1}(\xi, t)+\ldots
$$

Then, the double Mellin $G_{0}(N, M)$ satisfies eq. (3.2) with $\varphi=\chi_{0}$, while $G_{1}$ satisfies

$$
N G_{1}(N, M)=\widehat{\alpha}_{s}\left[\chi_{0}(M) G_{1}(N, M)+\chi_{1}(M) G_{0}(N, M)\right]+O\left(\widehat{\alpha}_{s}^{2}\right),
$$

where we have made use of the fact that the commutator of $\widehat{\alpha}_{s}$ and $\chi_{0}$ is of order $\widehat{\alpha}_{s}^{2}$. Eq. (3.23) is immediately recognized to have the same form as eq. (3.2), with a boundary condition now given by the $\chi_{1} G_{0}$ term. Hence, its solution has the form eq. (3.3) and factorization and duality are preserved. Specifically, $G_{1}$ is obtained multiplying the factorized $G_{0}$ eq. (3.21) by a suitable coefficient determined by $\chi_{1}$, and thus it has the structure of the standard perturbative solution, but now with the factor of $\alpha_{s}$ which multiplies $\chi_{1}$ promoted to running $\alpha_{s}(t)$. The procedure can then be pursued to higher orders: in fact, thanks to the expansion of $G$ in powers of $\alpha_{s}(t)$, the higher-order differential equation for $G$ which is found by simply letting $\alpha_{s} \rightarrow \widehat{\alpha}_{s}$ in the higher orders of the expansion of $\chi$ eq. (2.6) is reduced to a linear differential equation for each individual $G_{i}$.

Finally, it is interesting to observe that the inverse $N$-Mellin transform of $G(N, M)$ can actually be evaluated exactly if the boundary condition $I(N, M)$ is assumed to be 
a sufficiently smooth function of $N$, so that it may be effectively considered to be $N-$ independent, i.e. assuming that the boundary condition is $x$-independent, and all further $x$ dependence is generated by perturbative evolution. Indeed, using for definiteness eq. (3.21) and setting $I\left(N, t_{0}\right)=G_{00}$ one gets [34]

$$
G(\xi, M)=G_{00} \exp \frac{M-M_{0}}{\beta_{0} \alpha_{s}} I_{0}\left(2 \sqrt{\frac{\xi}{\beta_{0}} \int_{M_{0}}^{M} d M^{\prime} \varphi\left(M^{\prime}\right)}\right)
$$

where $I_{0}$ is the modified Bessel function. Even though it is not possible to determine the inverse $M$-Mellin transform of eq. (3.24) in closed form, the asymptotic behaviour of $G(\xi, t)$ when $t$ is large may be determined by performing the inverse $M$-Mellin by saddle point. The saddle condition is

$$
\sqrt{\frac{\beta_{0}}{\xi}} \frac{1}{\beta_{0} \alpha_{s}(t)}=\frac{I_{0}^{\prime}}{I_{0}} \frac{\varphi(M)}{\sqrt{\int_{M_{0}}^{M} d M^{\prime} \varphi\left(M^{\prime}\right)}}
$$

In the limit of large $\xi$, the left-hand side of eq. (3.25) vanishes, while on the right-hand side $I_{0}^{\prime} / I_{0} \underset{\xi \rightarrow \infty}{\sim} 1$ so the whole expression vanishes only if $\varphi$ goes through zero for some $M$ (recall that we assume $M \leq M_{0}$ in order for the perturbative limit to exist). Clearly if $\varphi$ is always positive, as is the case for $\varphi$ which has a minimum with a positive value, the saddle equation cannot be satisfied at a real value of $M$ : in such case, the asymptotic behaviour of $G(\xi, t)$ is dominated by a pair of complex saddle points, and therefore displays unphysical oscillations. Alternatively, if $\varphi$ has a vanishing or negative minimum, there is always a real saddle. The asymptotic limit is then stable, provided we choose $M_{0}$ equal to the value of $M$ where $\chi$ vanishes (otherwise, for negative minimum the square-root in eq. (3.24) becomes complex).

The onset of unphysical behaviour at large enough $\xi$ has been interpreted as a signal of breakdown of perturbation theory [21,28,30-32], and thus it has been viewed as an indication that the perturbative approach must be abandoned in the small $x$ limit. However, in the following section we will show that if we are interested in the BFKL approach as a tool to improve the theory of scaling violations at small $x$, then this negative conclusion is unjustified. Indeed, even when $G(\xi, t)$ determined by inverse Mellin from eq. (3.24) displays an asymptotic oscillatory behaviour, the associated splitting function remains smooth and monotonic, and therefore no oscillations are seen in the factorized solutions to leading-twist evolution equations.

\section{Quadratic Kernel and Asymptotic Behaviour}

In order to resum the running-coupling corrections to duality eq. (3.20) we must determine the parton distribution $G(N, t)$ by performing the inverse $M$ - Mellin transform in eq. (3.10) exactly, rather than in the saddle-point approximation as in the previous section. Even though it is nontrivial to do this in general, the integral can be easily 
determined $[27,25]$ if the kernel $\varphi$ in eq. (3.10) is quadratic. The ensuing form of the parton distribution displays the unphysical oscillations discussed at the end of the previous section. In this section we review this determination of $G(N, t)$, and then we use it to determine the running-coupling dual anomalous dimension eq. (3.20), and show that the corresponding splitting function, and thus the solution to the associated evolution equation, are free of oscillations and have a stable asymptotic $\xi \rightarrow \infty$ limit. In the next section we will then show that knowledge of the resummed anomalous dimension for a quadratic $\chi$ is sufficient to resum running-coupling singularities in general.

We start assuming that $\varphi$ is given by

$$
\varphi(M)=c+\frac{1}{2} \kappa\left(M-\frac{1}{2}\right)^{2}
$$

where $\kappa$ and $c$ are constants. In the sequel we will discuss the three cases of positive, negative, or vanishing $c$. While we will assume that $\kappa$ is positive but otherwise generic, for explicit numerical examples we will take

$$
\kappa=-\frac{2 C_{A}}{\pi} \psi^{\prime \prime}\left(\frac{1}{2}\right)
$$

(where $\psi(x)$ is the digamma function and $C_{A}=3$ ) which corresponds to the curvature of the leading-order BFKL kernel [6] $\chi_{0}$ (2.6) around its minimum. The expression of $G(N, t)$ is then given in terms of an Airy function [27]: inserting eq. (4.1) in eq. (3.21) and performing the $M$ integral with the choice $M_{0}=\frac{1}{2}$, we get

$$
G(N, t)=K(N) e^{1 /\left[2 \beta_{0} \alpha_{s}(t)\right]} \operatorname{Ai}\left[z\left(\alpha_{s}(t), N\right)\right] \frac{I\left(N, t_{0}\right)}{N}
$$

where $\operatorname{Ai}(z)$ is the Airy function, which satisfies

$$
\operatorname{Ai}^{\prime \prime}(z)-z \operatorname{Ai}(z)=0
$$

with $\operatorname{Ai}(0)=3^{-2 / 3} / \Gamma(2 / 3)$,

$$
z\left(\alpha_{s}(t), N\right) \equiv\left(\frac{2 \beta_{0} N}{\kappa}\right)^{1 / 3} \frac{1}{\beta_{0}}\left[\frac{1}{\alpha_{s}(t)}-\frac{c}{N}\right]
$$

and $K(N)$ is a $t$-independent normalization,

$$
K(N)=e^{-\left[\frac{1}{2 \beta_{0} \alpha_{s}}\right]}\left(\frac{2 \beta_{0} N}{\kappa}\right)^{1 / 3} \frac{1}{\pi} .
$$

Along the positive real axis, the Airy function is a positive definite, monotonically decreasing function of its argument, and it behaves asymptotically as

$$
\operatorname{Ai}(z)=\frac{1}{2} \pi^{-1 / 2} z^{-1 / 4} \exp \left(-\frac{2}{3} z^{3 / 2}\right)\left(1+O\left(z^{-3 / 2}\right)\right)
$$




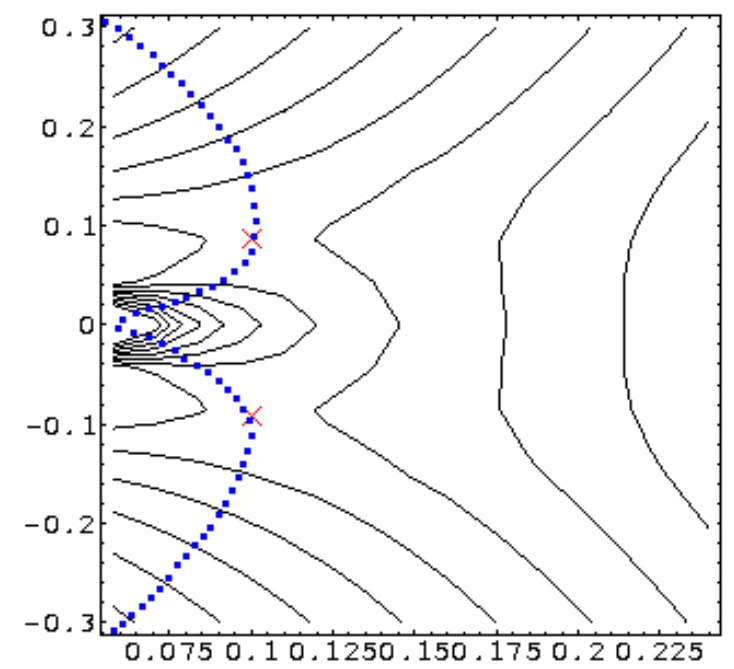

Figure 1: Contour plot in the complex $N$ plane for the Mellin inversion integral of $G(N, t)$. The crosses indicate the approximate location of the complex saddles, and the dotted line is the approximate steepest descent path.

For large enough $N, z \sim N^{1 / 3}$, so $\operatorname{Ai}\left[z\left(\alpha_{s}, N\right)\right]$ is also a monotonically decreasing function of $N$, asymptotically damped as $\operatorname{Ai}\left[z\left(\alpha_{s}, N\right)\right] \sim \exp -\sqrt{N}$. When $c=0$, this monotonic behaviour persists for all real positive $N$ even when $N$ is small. However, if $c>0, z$ changes sign when $N<\alpha_{s}(t) c$, and $\operatorname{Ai}(z)$ oscillates along the negative real $z$ axis, so $\operatorname{Ai}\left[z\left(\alpha_{s}, N\right)\right]$ oscillates as $N \rightarrow 0$, because $z \rightarrow-\infty$ in this limit. If instead $c<0, z \rightarrow \infty$ not only as $N \rightarrow \infty$ but also as $N \rightarrow 0$, and $\operatorname{Ai}\left[z\left(\alpha_{s}, N\right)\right]$ is smoothly damped in both limits.

It is now easy to check that the behaviour of $G(\xi, t)$ obtained by inverse Mellin transformation from $G(N, t)(4.3)$ has the asymptotic properties discussed in general at the end of sect. 3. Specifically, if $c$ (i.e. the value of $\chi(4.1)$ at its minimum) is positive, then $G(\xi, t)$ displays an unphysical oscillatory behaviour as $\xi \rightarrow \infty$. This can be seen explicitly by evaluating by saddle point the $N$-Mellin inversion integral which gives $G(N, t)$. The saddle point condition is dominated by the Airy function, and has thus the form

$$
\xi=-\frac{d \operatorname{Ai}\left[z\left(\alpha_{s}, N\right)\right] / d N}{\operatorname{Ai}\left[z\left(\alpha_{s}, N\right)\right]}
$$

If $\xi$ is not too large, the saddle condition is satisfied in the large $N$ region where $\operatorname{Ai}\left[z\left(\alpha_{s}, N\right)\right]$ is a decreasing function of $N$. As $\xi$ grows, the saddle is drawn towards $N \rightarrow 0$ where $\operatorname{Ai}\left[z\left(\alpha_{s}, N\right)\right]$ decreases faster with $N$; however, for sufficiently small $N$ the derivative changes sign and the real saddle is lost. This situation is displayed in fig. 1, where we show a contour plot in the complex $N$-plane of the real part of the integrand to the $N-$ Mellin inversion, namely $\operatorname{Re}[\xi N+\ln G(N, t)]$ with $G(N, t)$ given by eq. (4.3), $I\left(N, t_{0}\right)=1$, and $t=6$ (corresponding to $\alpha_{s}(t) \approx 0.2$ ), $\xi=10, c=1$. Along the real axis the function increases, and there is no saddle. However, even when $\xi \rightarrow \infty$ the saddle condition eq. (4.8) does still have a pair of complex solutions, with $|N| \rightarrow 0$ as $\xi$ grows. These saddle points can be clearly seen in fig. 1 .

For large $\xi$, the position of the complex saddle points can be determined explicitly 
using the asymptotic expansion eq. (4.7), which is valid when $|\arg (z)|<\pi$ : one finds

$$
N_{ \pm}=e^{ \pm i \pi / 4}(k / \xi)^{1 / 2}
$$

where $k \equiv \frac{2}{3} \frac{c}{\beta_{0}}\left(\frac{2 c}{\kappa}\right)^{1 / 2}$. The asymptotic large $\xi$ behaviour is dominated by the contributions from this pair of complex conjugate saddle points, which add to give cosine oscillations: explicitly

$$
G(\xi, t) \underset{\xi \rightarrow \infty}{\sim} \exp (\sqrt{2 k \xi}) \cos (\sqrt{2 k \xi}) .
$$

As $\xi$ becomes large, $G(\xi, t)$ becomes negative and starts to oscillate, so the solution looks unphysical.

If $c=0$, it is instead easy to see that eq. (4.8) has a solution for all $\xi$, with $N \rightarrow 0$ as $\xi \rightarrow \infty$. If, on the contrary, $c<0$ the real saddle is again lost as $N$ decreases because of the change of sign in the derivative of $d z / d N$. However, if one chooses $M_{0} \neq \frac{1}{2}$ the solution eq. (4.3) acquires an extra $N$-dependent prefactor, and it can be shown that one recovers a stable asymptotic behaviour if $M_{0}$ is chosen so that $\varphi\left(M_{0}\right)=0$. Hence, also in these cases one recovers the generic behaviours found from the Bessel function representation of $G(\xi, M)$ eq. (3.24).

At first sight the unphysical oscillatory behaviour of $G(N, t)$ when $c>0$ appears disastrous [21,27-32]. However, here we are not directly interested in the solution eq. (4.3), but rather in the dual anomalous dimension which can be extracted from it. This is given by

$$
\gamma_{A}\left(\alpha_{s}(t), N\right)=\frac{d}{d t} \ln G(N, t)=\frac{1}{2}+\left(\frac{2 \beta_{0} N}{\kappa}\right)^{1 / 3} \frac{\operatorname{Ai}^{\prime}\left[z\left(\alpha_{s}(t), N\right)\right]}{\operatorname{Ai}\left[z\left(\alpha_{s}(t), N\right)\right]},
$$

which we now study. The large $-N$ behaviour of $\gamma_{A}$ is easily determined, because when $N$ is large, $z$ is large and we can use the asymptotic expansion eq. (4.7) in eq. (4.11). This gives

$$
\gamma_{A}\left(\alpha_{s}, N\right) \sim \frac{1}{2}-\sqrt{\frac{2}{\kappa}\left[\frac{N}{\alpha_{s}}-c\right]}+O\left(\frac{1}{\left[z\left(\alpha_{s}, N\right)\right]^{3 / 2}}\right) .
$$

Note that this leading behaviour coincides with the 'naive dual' anomalous dimension: namely, the anomalous dimension which is found from $\varphi$ eq. (4.1) using the leading-order duality relation $(2.9)$, but with $\alpha_{s}=\alpha_{s}(t)$. We will come back to this point in the next section.

In order to determine the small- $N$ behaviour of $\gamma_{A}$ we must distinguish three cases. For positive values of $c$, as $N$ decreases $\gamma_{A}\left(\alpha_{s}(t), N\right)$ increases indefinitely until it blows up for the value $N=N_{0}(t)$ that corresponds to the first zero $z_{0} \approx-2.338 \ldots$ of $\operatorname{Ai}(z)$. Because $\mathrm{Ai}^{\prime}$ is regular at $z=z_{0}$, at $N=N_{0}$ the anomalous dimension has a simple pole. The location of this rightmost singularity of the anomalous dimension can be determined by solving the implicit equation $z\left[\alpha_{s}(t), N_{0}\right]=z_{0}$, with $z\left[\alpha_{s}(t), N_{0}\right]$ given by eq. (4.5). For small values of $\alpha_{s}(t)$ this solution can be expanded as

$$
N_{0}\left(\alpha_{s}, z\right)=c \alpha_{s}\left[1+\sigma+\frac{2}{3} \sigma^{2}+\frac{1}{3} \sigma^{3}+O\left(\sigma^{4}\right)\right]
$$




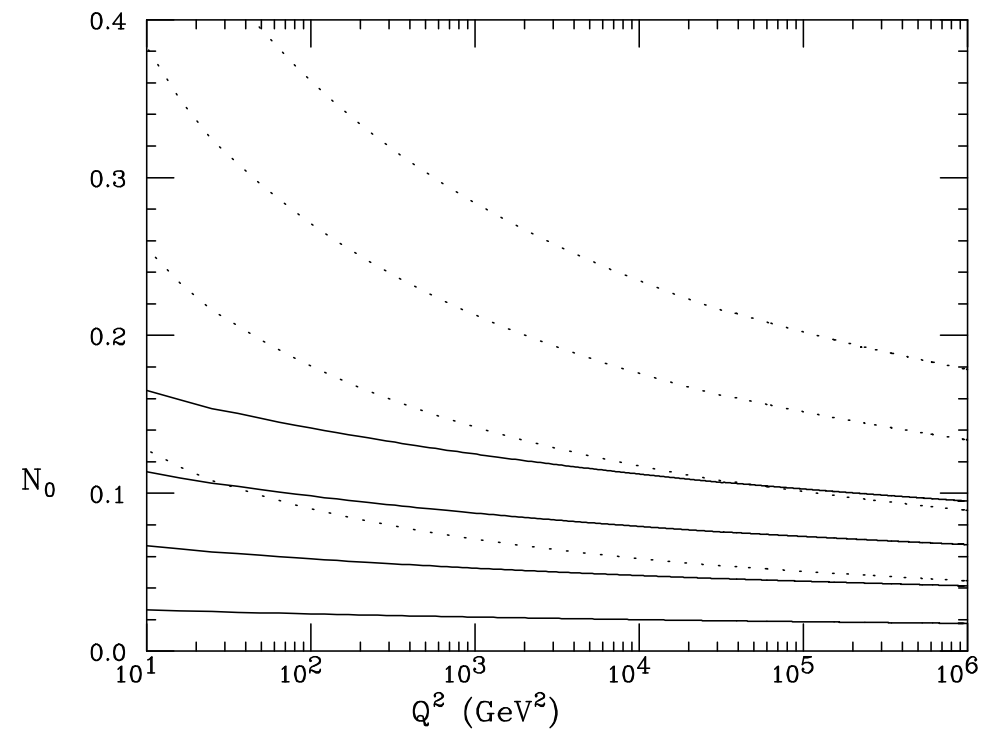

Figure 2: Location of the rightmost singularity of the Airy anomalous dimension (4.11), $N_{0}(t)$ (solid) and of the naive dual anomalous dimension (4.12), $\alpha_{s}(t) c$ (dotted). In both cases, the curves correspond (top to bottom) to $c=2,1.5,1,0.5$.

where

$$
\sigma\left(\alpha_{s}, z\right) \equiv\left(\beta_{0} \alpha_{s}\right)^{2 / 3}\left(\frac{\kappa}{2 c}\right)^{1 / 3}
$$

Recalling that $z \rightarrow-\infty$ as $N \rightarrow 0$ and $z_{0}<0$, eq. (4.13) implies that $N_{0}<\alpha_{s} c$. A plot of the position of the rightmost singularity $N_{0}(t)$ for several values of $c$ is displayed in fig. 2, along with the position of the rightmost singularity of the naive dual anomalous dimension eq. (4.12), namely the square-root branch cut at $N=\alpha_{s}(t) c .{ }^{3}$ Below $N_{0}$, as $N \rightarrow 0, z$ goes through an infinite number of zeros of the Airy function, and the anomalous dimension oscillates wildly. A plot of the anomalous dimension along the positive real axis (with $c=1$ ) is displayed in fig. 3: at small $N$ the anomalous dimension goes through an infinity of poles as $N \rightarrow 0$, while at large $\mathrm{N}$ the onset of the square-root drop eq. (4.12) is seen.

If $c \leq 0$, it is easy to see that $\operatorname{Ai}\left[z\left(\alpha_{s}(t), N\right)\right]$ does not have any zeros in the complex $N$ plane. Indeed, the zeros of $\operatorname{Ai}(z)$ are all located on the negative real $z$-axis. However, the cut induced by the function $z\left(\alpha_{s}(t), N\right)(4.5)$ must be taken along the negative $N$-axis, so that the $N$-Mellin inversion integral is well-defined in the physical region $N>0$. But the function $z\left(\alpha_{s}(t), N\right)$ then maps the cut $N$ plane on the quadrant $-\frac{2 \pi}{3} \leq z \leq \frac{2 \pi}{3}$ (if $c<0$ ) or $-\frac{\pi}{3} \leq z \leq \frac{\pi}{3}$ (if $c=0$ ). In either case, all the zeros of the Airy function lie outside the physical sheet of the $N$ plane. Furthermore, in both cases the anomalous dimension eq. (4.12) turns out to have a cut along the negative $N$ axis starting at $N=0$, which determines the small $x$ asymptotics.

Specifically, if $c=0$, then $z \propto N^{1 / 3}$ for all $N$. Because $\frac{\operatorname{Ai}^{\prime}(0)}{\operatorname{Ai}(0)}=-3^{1 / 3} \frac{\Gamma(2 / 3)}{\Gamma(1 / 3)}$ the

3 In this and subsequent figures we take the standard two-loop form of $\alpha_{s}(t)$, with $\alpha_{s}\left(M_{z}\right)=$ 0.119 . 


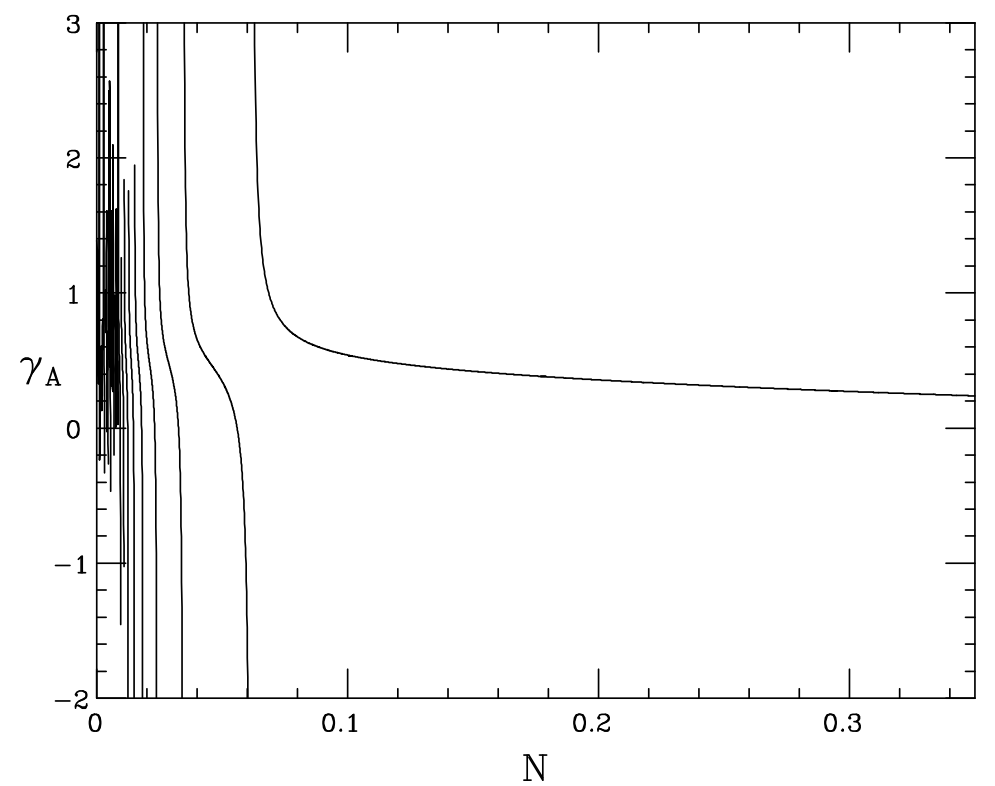

Figure 3: The Airy anomalous dimension $\gamma_{A}\left(\alpha_{s}, N\right)$ for $c=1$ and $\alpha_{s}=0.2$, showing the oscillations below the Airy zero at $N_{0}(t)$.

anomalous dimension has a cubic root branch cut at $N=0$ and is regular for $N>0$. If instead $c<0$, the cut can be seen by using the representation of the Airy function in terms of the modified Bessel functions $K_{\nu}$ to rewrite the anomalous dimension (4.12) as

$$
\gamma_{A}\left(\alpha_{s}, N\right)=\left(\frac{2\left(N-\alpha_{s} c\right)}{\kappa \alpha_{s}}\right)^{1 / 2} \frac{K_{2 / 3}\left(\frac{2}{3} z^{3 / 2}\right)}{K_{1 / 3}\left(\frac{2}{3} z^{3 / 2}\right)} .
$$

It is then easy to show using the continuation formula

$$
K_{\nu}(-x)=e^{-i \nu \pi} K_{\nu}(x)-i \pi I_{\nu}(x)
$$

that $\gamma_{A}$ has a discontinuity along the negative real $N$ axis, given by (as $|N| \rightarrow 0$ )

$$
\operatorname{disc}\left(\gamma_{A}\right)=-2 i\left(\frac{-2 c}{\kappa}\right)^{1 / 2} \exp -\left[\frac{4}{3} \frac{1}{\beta_{0}|N|} \sqrt{\frac{-2 c^{3}}{\kappa}}\right]+O(N) .
$$

Notice that if $c<0$, then when $N$ is small, $z$ is large and the expansion eq. (4.12) holds. This would seem to suggest that the anomalous dimension is regular and positive at $N=0$. However, this conclusion cannot be drawn because this expansion is merely asymptotic: in fact, it is factorially divergent, though Borel summable. Its Borel sum coincdes with eq. (4.15), with the discontinuity eq. (4.17), which is exponentially subleading and thus not immediately visible in the asymptotic expansion eq. (4.12).

We can now study the splitting function $P_{A}$, related by Mellin transform eq. (2.5) to the Airy anomalous dimension $\gamma_{A}\left(\alpha_{s}(t), N\right)$. If $c>0$, the asymptotic $\xi \rightarrow \infty$ behaviour of the splitting function is dominated by the pole at $N=N_{0}(t)$, which is the rightmost singularity of the anomalous dimension:

$$
x P_{A}\left(\alpha_{s}(t), x\right) \underset{x \rightarrow 0}{\sim} \alpha_{s}(t) x^{-N_{0}(t)} .
$$




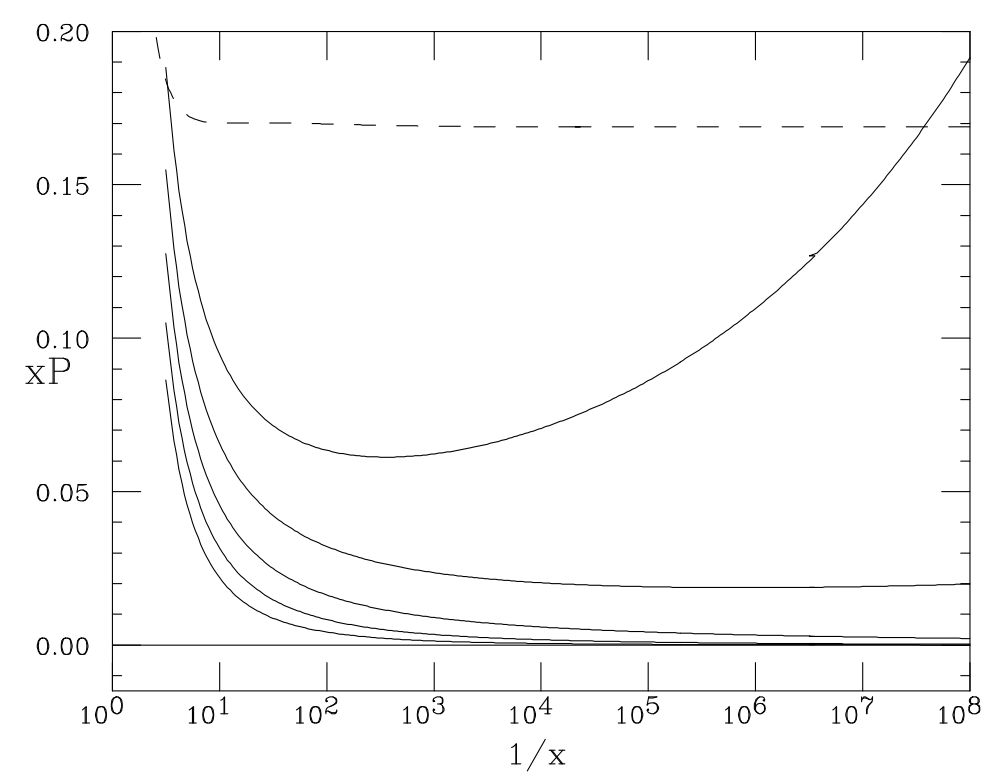

Figure 4: The Airy splitting function $P_{A}\left(\alpha_{s}, x\right)$ with $\alpha_{s}=0.2$, and (top to bottom) $c=2,1,0,-1,-2$, showing the absence of oscillations at very small $x$ (solid). The standard two-loop splitting function is also shown (dashed).

If $c \leq 0$, the small $x$ behaviour is instead controlled by the cut along the negative $N$ axis. Specifically, if $c=0$ branch cut is cubic, and the asymptotic behaviour can be estimated noting that the inverse Mellin of $N^{1 / 3}$ is proportional to a gamma function: consequently when $c=0$

$$
x P_{A}\left(\alpha_{s}(t), x\right) \underset{x \rightarrow 0}{\sim} \frac{1}{\xi^{4 / 3}} .
$$

If instead $c<0$ the asymptotic behaviour can be estimated by evaluating by saddle point the integral along the discontinuity eq. (4.17), with the result

$$
x P_{A}\left(\alpha_{s}(t), x\right) \underset{x \rightarrow 0}{\sim} \exp \left[-\frac{4}{\sqrt{3}}\left(\frac{-2 c^{3}}{\kappa}\right)^{1 / 4}\left(\frac{\xi}{\beta_{0}}\right)^{1 / 2}\right] .
$$

Therefore, asymptotically if $c>0$ the splitting function grows as an inverse power of $x$, if $c=0$ it drops as a power of $\xi=\ln (1 / x)$, and if $c<0$ it drops faster than any power of $\xi$ but slower than a power of $x$. Whatever the value of $c$, the splitting function is smooth for all $\xi>0$. A plot of $x P_{A}\left(\alpha_{s}(t), x\right)$ for $c=2,1,0,-1,-2$ is displayed in fig. 4, along with the usual two-loop splitting function: it is apparent that the splitting function is well-defined and positive for arbitrarily small $x$. The fact that the anomalous dimension has singularities for $N \leq N_{0}(t)$ (if $c>0$ ) only means that $N_{0}(t)$ is the lowest moment of the splitting function for which the integral eq. (2.5) over $x$ converges, but does not imply that there are problems in the $t$-evolution. The effect of the running of the coupling is to replace the naive dual anomalous dimension eq. (4.12) with the resummed anomalous dimension eq. (4.11). We can then equivalently view the effect of the running of the coupling as a modification of the evolution kernel, whereby the duality relation (2.9) 


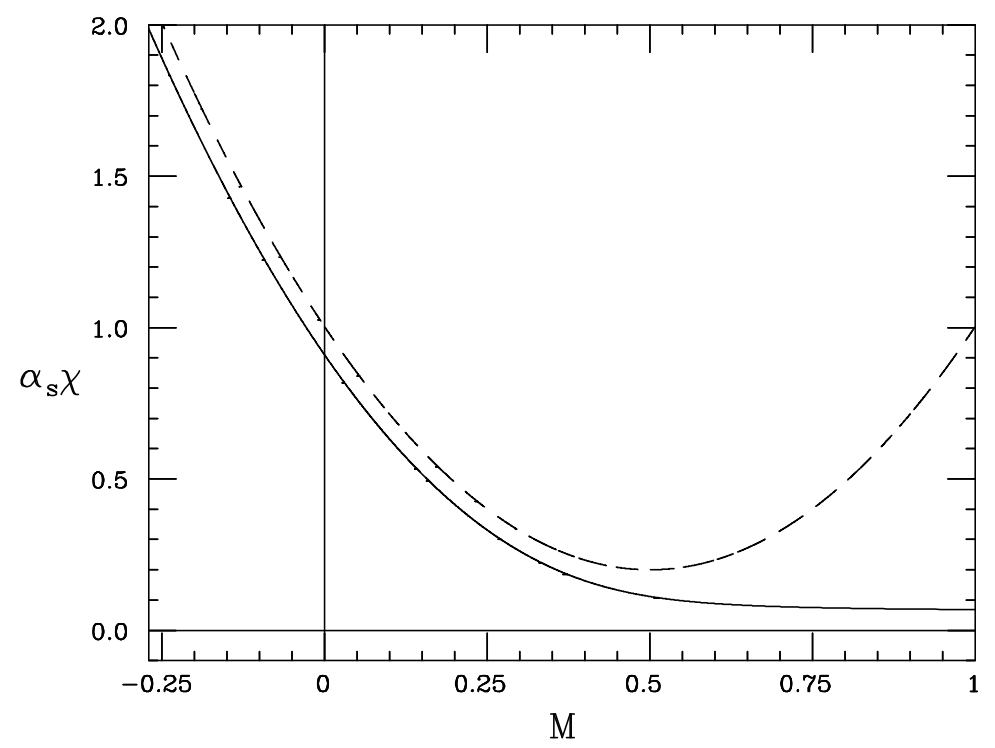

Figure 5: The kernel $\varphi(M)$ eq. (4.1) (dashed) and the Airy effective kernel $\chi_{A}(M)$ eq. (4.21) (solid) with $c=1$ and $\alpha_{s}=0.2$.

is preserved, but the 'bare' kernel $\varphi(M)$ (4.1) is replaced by a resummed Airy effective kernel, defined by the duality equation

$$
\alpha_{s} \chi_{A}\left(\gamma_{A}\left(\alpha_{s}, N\right)\right)=N
$$

in terms of the Airy anomalous dimension $\gamma_{A}\left(\alpha_{s}, N\right)$ (4.11). In the next section we will show explicitly that the perturbative effective kernel eq. (2.17) is an (asymptotic) expansion of the Airy effective kernel eq. (4.21). The bare and effective kernel (when $c=1$ ) are compared in fig. 5. Note that indeed at small $M$ (i.e., by duality, large $N$ ) the resummed and naive kernel coincide, up to a subleading (asymptotically constant) correction, in agreement with eq. (4.12).

Because the resummed splitting function is smooth, the solution to perturbative evolution driven by it is also smooth and free of oscillations. This can be understood by noting that the solution of the evolution equation (2.3) with the anomalous dimension eq. (4.11) and the boundary condition $G\left(N, t_{0}\right)=\tilde{F}_{0}(N)$ is

$$
\begin{aligned}
G(N, t) & =\Gamma\left(t, t_{0} ; N\right) \tilde{F}_{0}(N), \\
\Gamma\left(t, t_{0} ; N\right) & \equiv e^{\int_{t_{0}}^{t} \gamma_{A}\left(\alpha_{s}(t), N\right) d t}=\frac{G(N, t)}{G\left(N, t_{0}\right)},
\end{aligned}
$$

with the evolution factor $\Gamma\left(t, t_{0} ; N\right)$ explicitly given by

$$
\Gamma\left(t, t_{0} ; N\right)=e^{1 /\left[2 \beta_{0} \alpha_{s}(t)\right]-1 /\left[2 \beta_{0} \alpha_{s}\left(t_{0}\right)\right]} \frac{\operatorname{Ai}\left[z\left(\alpha_{s}(t), N\right)\right]}{\operatorname{Ai}\left[z\left(\alpha_{s}\left(t_{0}\right), N\right)\right]} .
$$

Now, for large enough $\xi$, the oscillations eq. (4.10) are the same at all values of $t$, and thus cancel in the ratio $G(N, t) / G\left(N, t_{0}\right)$. This is a consequence of the fact that the saddle 


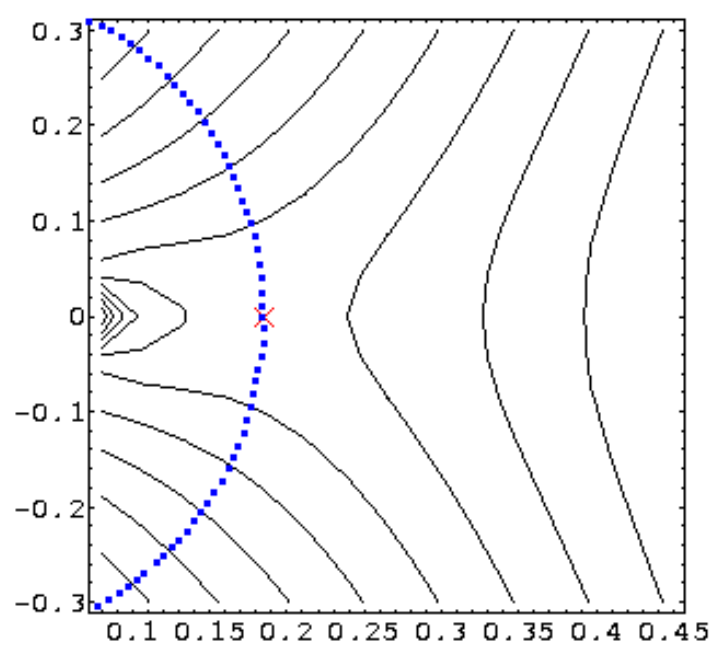

Figure 6: Contour plot in the complex $N$ plane for the Mellin inversion integral of $G(N, t) / G\left(N, t_{0}\right)$. The cross indicates the approximate location of the real saddle, and the dotted line is the approximate steepest descent path.

points which drive the large $\xi$ oscillations are dominated by the $t$-independent $-c / N$ contribution to $z(4.5)$, which at small $N$ overwhelms the $1 / \alpha_{s}(t)$ term.

The effect of this $t$-factorisation is apparent comparing the contour plot of fig. 1 with fig. 6, where we display $\operatorname{Re}\left[\xi N+\ln \left(G(N, t) / G\left(N, t_{0}\right)\right)\right]$ (all the other parameters being as in fig. 1). In this case there is a saddle point on the real axis, while the complex saddle points have disappeared. The steepest descent contour goes over the real saddle, where the integrand is real, and there are no oscillations. In other words, whenever oscillations are present they are factorized in the initial condition, while the evolution factor $\Gamma\left(t, t_{0} ; N\right)(4.22)$ has an inverse Mellin transform which is smooth and asymptotically monotonic in $\xi$ as $\xi \rightarrow \infty$.

In conclusion, in order to understand the impact of the running coupling resummation, we give some estimates of the asymptotic behaviour of the inverse $N$-Mellin transform of the evolution factor $\Gamma\left(t, t_{0} ; N\right)$, i.e. of the solution $G(N, t)$ eq. (4.22) when the boundary condition $\tilde{F}_{0}(N, t)$ is constant. If $c>0$, the asymptotic behaviour of the evolution factor (4.23) is dominated by its rightmost singularity, which is the simple pole at $N=N_{0}\left(t_{0}\right)$, where the Airy function in the denominator vanishes. The asymptotic behaviour is then the same as that of the splitting function eq. (4.19):

$$
G(\xi, t) \sim \alpha_{s}\left(t_{0}\right) x^{-N_{0}\left(t_{0}\right)} .
$$

Note that because $N_{0}(t)<\alpha_{s}(t) c$ the behaviour of the resummed evolution factor is significantly softer than that which is found using the naive dual anomalous dimension eq. (4.12), namely $G \sim x^{-\alpha_{s} c}$ (see fig. 2). The same result is of course found by dominating the integral with the saddle point displayed in fig. 6 .

If $c=0$ the rightmost singularity of the evolution factor is a branch cut at $N=0$. In this case, the asymptotic behaviour is dominated by a saddle point, located on the positive 
real axis at

$$
N_{s}=\left(\frac{k\left(t-t_{0}\right)}{\xi}\right)^{3 / 2}
$$

with $k=\Gamma(2 / 3) / \Gamma(1 / 3) 3^{-2 / 3}\left(2 \beta_{0} / \kappa\right)^{1 / 3}$. This leads to

$$
G(\xi, t) \underset{\xi \rightarrow \infty}{\sim} \frac{1}{\sqrt{\xi^{5 / 2} \alpha_{s}(t)^{3 / 2}}} \exp \left[\frac{k\left(t-t_{0}\right)^{3 / 2}}{\xi^{1 / 2}}\right] .
$$

Because the leading asymptotics is constant, in this case we have also given the first subleading correction, which corresponds to a power drop in $\xi$. This drop is somewhat milder than the $\xi^{-3 / 2}$ drop which is found [23] using the naive dual anomalous dimension eq. (4.12).

Finally, if $c<0$ the behaviour can be estimated using a procedure analogous to that which led to the corresponding behaviour of the splitting function, eq. (4.20), and in fact it turns out to be the same:

$$
G(\xi, t) \underset{\xi \rightarrow \infty}{\sim} \exp \left[-\frac{4}{\sqrt{3}}\left(\frac{-2 c^{3}}{\kappa}\right)^{1 / 4}\left(\frac{\xi}{\beta_{0}}\right)^{1 / 2}\right]
$$

Notice that this asymptotic behaviour is subdominant in comparison to that induced by a perturbative $N=0$ pole, such as is present in the standard one loop anomalous dimension. In fact, if the boundary condition to perturbative evolution has a $N=0$ pole (corresponding to a constant behaviour in $x$ ), the asymptotic behaviour of the evolution factor eq. (4.24) for $N_{0}<0$ will be subdominant to that of the boundary condition. It is, however, a slower drop in comparison to the power drop $G \sim x^{-\alpha_{s}(t) c}$ which would be found using the naive dual anomalous dimension eq. (4.12)

\section{Asymptotic Behaviour and Resummation: the Generic Case}

The solution of the running coupling $\xi$-evolution equation with a quadratic kernel studied in the previous section gives the small $x$ asymptotic behaviour for generic evolution kernels $\chi$ which have a minimum in the physical region $0 \leq M \leq 1$. Consequently, it is possible to use this solution to resum the perturbative running-coupling singularities.

The key observation which allows one to exploit the results of the Airy resummation is that the asymptotic small- $x$ behaviour of the solution is dominated by the form of the kernel in the neighbourhood of its minimum, so we can view the quadratic kernel eq. (4.1) as the low-order truncation of the Taylor expansion of the full kernel about its minimum. Indeed, it was proven in ref. [23] that if the running is included to finite perturbative order (or not at all), then the coefficient of the leading behaviour in the splitting function at each order is entirely determined by the value of the kernel and its second derivative at the minimum, while higher-order derivatives only affects terms which are asymptotically suppressed by inverse powers of $\xi$. 
It is easy to check that this remains true after resummation of the running coupling effects (at least if $c \geq 0$ ). To see this, assume that the kernel eq. (4.1) is corrected by a cubic term:

$$
\varphi(M)=c+\frac{1}{2} \kappa\left(M-\frac{1}{2}\right)^{2}+\frac{1}{6} \epsilon\left(M-\frac{1}{2}\right)^{3} .
$$

The solution eq. (3.21) can then be written as

$$
G(N, t)=e^{-\frac{1}{6} \epsilon \frac{d^{3}}{d t^{3}}} G^{(2)}(N, t),
$$

where $G^{(2)}(N, t)$ is the solution which corresponds to a quadratic kernel $\varphi(M)$, given by eq. (4.3). The fact that the cubic term only leads to a subleading correction to the asymptotic behaviour is then a direct consequence of the fact that

$$
\frac{d}{d t}=\left(\frac{2 \beta_{0} N}{\kappa}\right)^{1 / 3} \frac{d}{d z} .
$$

Indeed, assume that the cubic term is treated perturbatively. The change of asymptotic behaviour induced by this term is then due to the fact that the position $z_{0}$ of the rightmost zero of the Airy solution is shifted by the correction. The shifted zero is located at $z_{0}+\delta$, where, expanding eq. (5.3), $\delta$ to first order is given by

$$
\delta=\frac{1}{6} \epsilon \frac{2 \beta_{0} N}{\kappa} \frac{\operatorname{Ai}^{\prime \prime \prime}\left(z_{0}\right)}{\operatorname{Ai}^{\prime}\left(z_{0}\right)} .
$$

Hence, $\delta$ is proportional to $N$ and thus suppressed by inverse powers of $\xi$ in the $x \rightarrow 0$ limit, and so the leading behaviour of the splitting function and solution as $x \rightarrow$ 0 are still asymptotically given by $x^{-N_{0}(t)}$, as in the quadratic case eqs. (4.18),(4.24). A similar line of argument leads to the general conclusion that the leading asymptotic behaviour is not affected by corrections to the quadratic approximation, although of course subleading corrections to it are affected, as long as $c \geq 0$. If $c<0$ these arguments fail, because the asymptotic behaviour is dominated by the neighbourhood of the point $N \sim 0$ (see eq. (4.20)) i.e. (by duality) the neighbourhood of $M_{0}$ such $\chi(M)=0$, and not by the minimum of $\chi$. However in this case running coupling effects are subdominant in comparison to the low-order perturbative terms and the boundary condition, (compare eq. (4.27)) and thus of no concern.

We can now exploit the universality of the quadratic result to resum running coupling singularities, by matching the Airy anomalous dimension to the singular anomalous dimension obtained at NLLx. The first step in the procedure is to derive an expansion of the Airy anomalous dimension (4.12) of the form eq. (3.20), namely, an expansion in powers of $\alpha_{s}(t)$ at fixed $\alpha_{s}(t) / N$. Because

$$
\left[z\left(\alpha_{s}, N\right)\right]^{-3 / 2}=\frac{1}{\sqrt{\frac{2}{\kappa}\left(\frac{N}{\alpha_{s}}-c\right)}} \beta_{0} \alpha_{s} \frac{1}{\left(1-\frac{\alpha_{s} c}{N}\right)},
$$


the large $z$ asymptotic formula in eq. (4.7) can be used to this purpose. The leading order term, already given in eq. (4.12), is now recognized as the leading order of the duality expansion (3.20) of the Airy anomalous dimension:

$$
\gamma_{A}\left(\alpha_{s}, N\right)=\frac{1}{2}-\sqrt{\frac{2}{\kappa}\left[\frac{N}{\alpha_{s}}-c\right]}-\frac{1}{4} \frac{\beta_{0} \alpha_{s}}{1-\frac{\alpha_{s}}{N} c}+O\left(\alpha_{s}^{2}\right) .
$$

So the leading order term has the form that corresponds to $\varphi$ in eq. (4.1) by the duality relation eq. (2.9). The term of order $\alpha_{s} \beta_{0}$ in eq. (5.6) is the lowest order effect of running: indeed, it is equal to $\Delta \gamma_{s s}$ eq. (2.16),(3.18) for the quadratic $\varphi$ of eq. (4.1). In fact, the asymptotic expansion eq. (4.7) coincides with the asymptotic expansion in powers of $N \beta_{0} / \varphi^{\prime 3}$ eq. (3.19), obtained by evaluating by saddle-point the $M$-Mellin integral, so the expansion of the Airy function eq. (3.20) is recognized to be the same as the runningcoupling duality expansion of the anomalous dimension eq. (3.20), for the particular case of a quadratic $\chi$ kernel. Hence, the perturbative effective kernel eq. (2.17) is an expansion of the Airy effective kernel eq. (4.21). The expansion is factorially divergent but Borel summable.

Furthermore, the universality of the quadratic asymptotic behaviour implies that the coefficient of the leading running-coupling singularity to each order is entirely determined by knowledge of the value of $\chi$ and its second derivative at the minimum. This can be seen explicitly at NLLx by considering the running-coupling correction to the dual kernel $\Delta \chi_{1}$ eq. (2.18): whereas of course the function $\Delta \chi_{1}(M)$ for generic $M$ is determined by the full $\varphi$, the residue of the pole at $M=\frac{1}{2}$ is entirely determined by knowledge of $c$, viewing eq. (4.1) as the quadratic truncation of the Taylor series of $\chi$ about $M=\frac{1}{2}$ :

$$
\Delta \chi_{1}=\frac{\beta_{0}}{2} \frac{c}{M-\frac{1}{2}}+O\left(M-\frac{1}{2}\right) .
$$

Therefore, the Airy effective kernel eq. (4.21) is in fact a (Borel) resummation of the leading running coupling corections to the kernel eq. (2.17) for generic kernels with a minimum, and not only in the particular case of a quadratic kernel.

It follows that we may resum the running coupling singularities $\gamma_{\mathrm{rc}}$ eq. (3.20) by adding to the anomalous dimension $\gamma$ the Airy anomalous dimension which corresponds to the quadratic approximation to the kernel $\chi$ which is dual to $\gamma$, and subtracting off the NLLx expansion eq. (4.12) in order to avoid double counting. To NLLx it is in fact sufficient to determine the Airy resummation from the $\chi$-kernel which is dual to the leading anomalous dimension $\gamma_{s}$ : explicitly, to this order

$$
\begin{aligned}
\gamma_{\mathrm{res}}\left(\alpha_{s}, N\right)=\gamma_{s}\left(\frac{\alpha_{s}}{N}\right)+\alpha_{s} \gamma_{s s}\left(\frac{\alpha_{s}}{N}\right)+\alpha_{s} \Delta \gamma_{s s}\left(\frac{\alpha_{s}}{N}\right) \\
+\gamma_{A}\left(\alpha_{s}, N\right)-\frac{1}{2}+\sqrt{\frac{2}{\kappa \alpha_{s}}\left[N-\alpha_{s} c\right]}+\frac{1}{4} \frac{\beta_{0} \alpha_{s} N}{N-\alpha_{s} c}
\end{aligned}
$$

where $c$ is the value at its minimum of the $\chi$-function dual to $\gamma_{s}$. Using eq. (5.7) and duality, the last term is seen to cancel the singularity in $\Delta \gamma_{s s}\left(\frac{\alpha_{s}}{N}\right)$, as it must since the singularities have been resummed in the Airy anomalous dimension $\gamma_{A}$ eq. (4.11). 


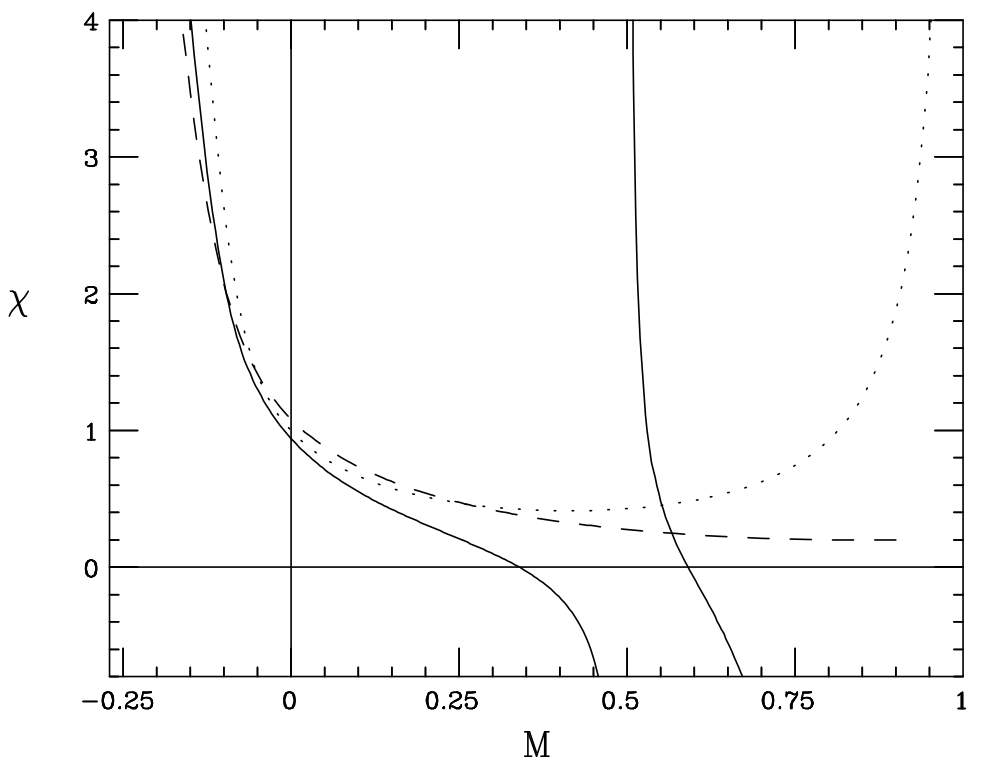

Figure 7: The leading- (dotted) and next-to-leading order (solid) double-leading $\chi$ kernels compared to the resummed effective kernel (dashed). All curves are computed with $\alpha_{s}=0.2$ in the $\mathrm{R}$-resummation scheme of ref. [20].

The resummed anomalous dimension $\gamma_{\text {res }}$ eq. (5.8) coincides with the unresummed one up to terms which are $\mathrm{N}^{2} \mathrm{LLx}$, so the resummation does not change the known low-order behaviour of the anomalous dimension. In fact, $\gamma_{A}$ is rather small in comparison to the standard two-loop anomalous dimension (compare fig. 4), thus it only gives a rather small contribution to $\gamma_{\mathrm{res}}\left(\alpha_{s}, N\right)$ eq. (5.8): the main effect of the resummation procedure is the subtraction of the running coupling singularities. At asymptotically small $x$, the leading behaviour of the unresummed $\gamma_{s}$ and $\gamma_{s s}, x^{-\alpha_{s} c}$, is replaced by the resummed behaviour $\left(x^{-N_{0}}\right.$, if $\left.c>0\right)$. However the subleading unresummed behaviour is not affected by the resummation procedure. The subleading behaviour, due to terms in the BFKL kernel beyond the quadratic approximation, is suppressed by powers of $\xi$ in comparison to the leading one, i.e. it is of the form $\frac{1}{\xi^{k}} x^{-\alpha_{s} c}$. If these terms were resummed, the subleading $\frac{1}{\xi^{k}} x^{-\alpha_{s} c}$ behaviour would be also replaced by a resummed one, $x^{-N_{0}+\delta(\xi)}$, with $\delta \sim \xi^{-n}$, as in eq. (5.4). This further subleading running-coupling resummation is lacking in eq. (5.8), and it will become necessary when $x$ is so small that the shape of the anomalous dimension around $N \sim \alpha_{s} c$ is important.

By using the resummed anomalous dimension eq. (5.8) in the duality relation eq. (2.9), we may finally determine a resummed effective $\chi_{\text {eff }}$ eq. (2.17). The effect of this resummation is shown in fig. 7 , where we compare the effective $\chi$ determined at leading and next-to-leading order of the double-leading expansion [19,20], and the resummed nextto-leading result. The NLO kernel is affected by the singularity, which is then removed by the resummation. Note that the resummed result shown in this figure is defined as the dual (2.9) of the resummed $\gamma$, obtained by combining the Airy resummation eq. (5.8) with the double-leading expansion of the anomalous which we discussed in refs. [19,20] (see fig. 8 below). For ease of comparison with our previous work, the double-leading kernel shown here is the same as that displayed in fig. 2 of ref. [19], which violates the momentum 
conservation constraint $\chi(0)=1$ by sub-subleading terms. The resummation procedure is then applied to this kernel and thus leads to a result which also does not exactly conserve momentum.

In $\overline{\mathrm{MS}}$-like schemes the running coupling singularities are actually shifted into the quark sector, so the resummation should instead be performed in the quark sector. This can be done by noting that the running coupling singularities can be removed from the anomalous dimension $\gamma^{+}$by redefining the normalization of the gluon distribution by a factor $u\left[\gamma_{s}\left(\alpha_{s} / N\right)\right]$. Such a redefinition amounts to a conventional change of factorization scheme provided only that $u[\gamma(0)]=1[33,20]$. Upon this redefinition the large eigenvalue of the anomalous dimension matrix $\gamma^{+}$(which so far we identified with the anomalous dimension, recall eq. (2.1)) and the quark sector anomalous dimension $\gamma_{q g}$ change according to $[33,20]$

$$
\begin{aligned}
& \gamma^{+} \rightarrow{\gamma^{+}}^{\prime}=\gamma^{+}+\left.\beta_{0} \frac{\chi_{0}\left(\gamma_{s}\right)}{\chi_{0}^{\prime}\left(\gamma_{s}\right)} \frac{d}{d M}[\ln u(M)]\right|_{M=\gamma_{s}} \\
& \gamma^{q g} \rightarrow \gamma^{q g^{\prime}}=u^{-1}\left(\gamma_{s}\right) \gamma^{q g} .
\end{aligned}
$$

The choice $u\left(\gamma_{s}\right)=\frac{\sqrt{-\chi_{0}^{\prime}\left(\gamma_{s}\right)}}{N / \alpha_{s}}$ is then sufficient to remove the singular contribution eq. (2.16) from $\gamma^{+}$(while the factor in the denominator ensures that this is a bona fide scheme change). The scheme change, however, introduces a square-root singularity in the quark anomalous dimension $\gamma^{q g}$ : indeed, we get

$$
\begin{aligned}
& \gamma^{+^{\prime}}=\gamma^{+}+\beta_{0}\left(\frac{\chi_{0}^{\prime \prime}\left(\gamma_{s}\right) \chi_{0}\left(\gamma_{s}\right)}{2 \chi_{0}^{\prime 2}\left(\gamma_{s}\right)}-1\right) \\
& \gamma^{q g^{\prime}}=\frac{\chi_{0}\left(\gamma_{s}\right)}{\sqrt{-\chi_{0}^{\prime}\left(\gamma_{s}\right)}} \gamma^{q g} .
\end{aligned}
$$

The transformation which takes one to the $\overline{\mathrm{MS}}$ scheme [7] is instead given by a factor $u(M)=R(M)=r(M) / \sqrt{\chi_{0}^{\prime}}$, where $r(M)$ is a regular function of $M$. Now, we can perform the desired running coupling resummation in the quark sector by starting with the resummed anomalous dimension eq. (5.8), and then performing a scheme change of the form eq. (5.9), while demanding that $\gamma^{+^{\prime}}$ be still given by eq. (5.10). The resummation is thus shifted entirely into the quark sector, and $\gamma^{q g^{\prime}}$ is no longer given by eq. (5.11), but rather by

$$
\gamma^{q g^{\prime}}\left(\alpha_{s}, N\right)=\operatorname{Ai}\left[z\left(\alpha_{s}(t), N\right)\right] e^{\frac{2}{3}\left[z\left(\alpha_{s}, N\right)\right]^{-3 / 2}} \gamma^{q g}\left(\alpha_{s}, N\right),
$$

which shows explicitly that the square-root singularity in $\gamma^{q g^{\prime}}$ eq. (5.11) is eliminated by the resummation (the extra exponential factor removes the term corresponding to $\gamma_{s}$ in the expansion of the Airy resummed contribution).

This running-coupling resummation can finally be combined with the small- $x$ resummation of the anomalous dimension previously discussed by us $[19,20]$. In such case, the expansion of the anomalous dimension in powers of $\alpha_{s}$ at fixed $\alpha_{s} / N$ is obtained by duality from a reorganized expansion of the $\chi$ kernel, where in particular the leading-order term is

$$
\alpha_{s} \tilde{\chi}_{0}=\alpha_{s} \chi_{0}+\Delta \lambda
$$




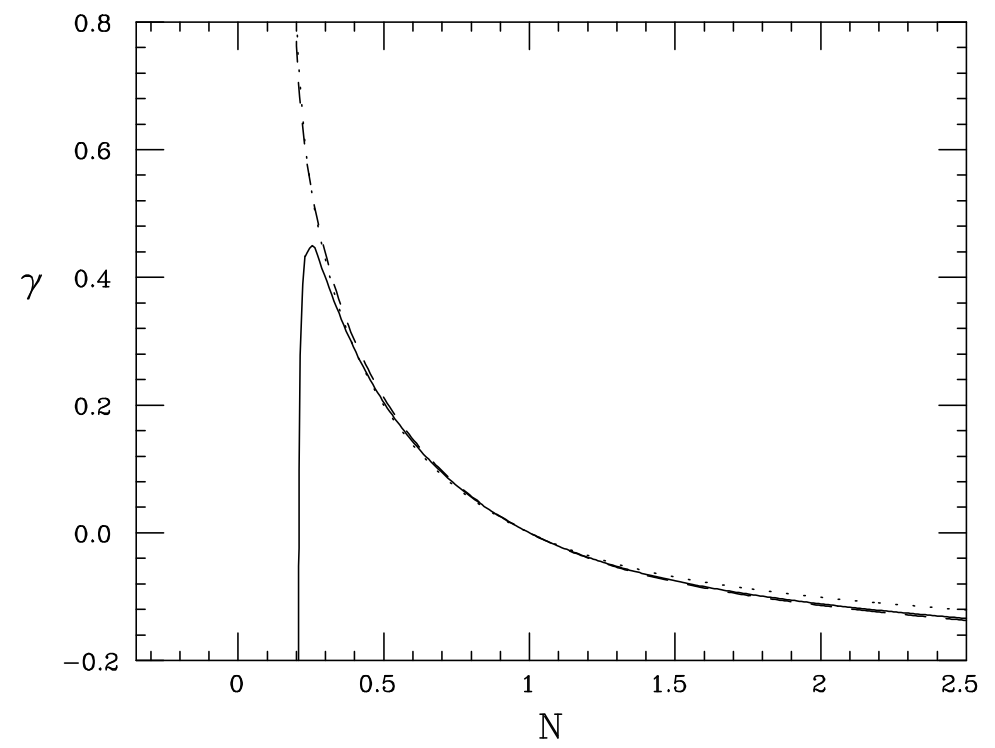

Figure 8: The double-leading anomalous dimensions with $c=1$ and $\alpha_{s}=0.2$ (i.e. $\lambda=0.2[20])$ computed to NLO in the R-resummation scheme of ref. [20] with $\overline{\mathrm{MS}}$ factorization (dotted). The same after the scheme change eq. (5.10) (solid), displaying the running-coupling singularity, and then after the singularity is removed by resummation (5.8) (dashed).

with $\Delta \lambda=\alpha_{s}^{2} \chi_{1}(1 / 2)+\ldots$ such that $\lambda \equiv \alpha_{s} \chi_{0}(1 / 2)+\Delta \lambda$ coincides with the value of the all-order $\chi$ at its minimum. Consequently, $\lambda$ must be treated as a free parameter. To resum the corresponding NLLx running coupling singularity it is sufficient to identify the quadratic $\varphi$ eq. (4.1) with the expansion of $\tilde{\chi}_{0}$ about its minimum, so that $c=\lambda / \alpha_{s}$, while $\kappa$ is given by eq. (4.2), and then resum according to eq. (5.8). This resummed NLLx anomalous dimension can then be combined with the standard two-loop anomalous dimension $[19,20]$ to give a fully resummed double-leading expansion.

The phenomenological impact of these manipulations can be understood on the basis of the asymptotic estimates discussed in the previous section. If $c>0$ the running coupling resummation shifts the rightmost singularity of the small $-x$ anomalous dimension from $N=\alpha_{s}(t) c$ to the smaller value $N=N_{0}(t): N_{0}(t)<\alpha_{s}(t) c$, as discussed in the previous section (see eq. (4.13)). The asymptotic behaviour is thus somewhat softened. If $c=0$ the running coupling resummation has a modest effect, in that it simply turns the square-root branch point of the unresummed naive dual anomalous dimension eq. (4.12) into a cubic branch point; in fact, when $c=0$ there are no running coupling singularities, since in this case $\Delta \chi_{1}(2.18)$ and its higher-order generalizations are regular as $M \rightarrow \frac{1}{2}$ [20]. Finally, if $c<0$, both the unresummed and resummed behaviours are subdominant in comparison to the perturbative behaviour, and thus the phenomenological impact of the resummation is negligible.

This situation is displayed in fig. 8, with the phenomenologically plausible [20] value $c=1$. In this figure, we show the NLO double-leading anomalous dimension $\gamma^{+}$in the $\overline{\mathrm{MS}}$ scheme (used in ref. [19]), and we compare it to the asymptotically unstable anomalous dimension obtained from it performing in reverse the scheme change eq. (5.10), so the 
running coupling singularity is moved back into $\gamma^{+}$. Note that the momentum conservation constraint $\gamma^{+}(1)=0$ is exactly enforced on all the anomalous dimensions shown in fig. 8, by means of a sub-subleading subtraction, as discussed in ref. [19]. The scheme change has a very small effect for almost all values of $N$, but it generates a strong instability in a small region of $N, 0.2<N \lesssim 0.23$. (recall that the anomalous dimension has a branch cut at $N=\lambda$ ). Finally, we show the result of applying the resummation eq. (5.8) to this unstable anomalous dimension: the instability is completely removed, and the anomalous dimension remains smooth for all $N>\lambda$. The softening of the small $N$ behaviour in the vicinity of the branch cut appears to be marginal, as expected because of the smallness of the $\gamma_{A}$ contribution to the resummed anomalous dimension eq. (5.8)(see fig. 4). Hence, the main effect of the resummation is to remove the instability, thereby widening the region of validity of the resummed anomalous dimension down to $N=\lambda$. Below this value, a further subleading running-coupling resummation would be required in order to recover the correct asymptotic $x^{-N_{0}}$ behaviour. The phenomenological effects of the instability and its resummation turn out to be small in the HERA region because of the limited range of $N$ in which it appears, but they may become relevant at yet higher energies [20].

The running coupling resummation can be generalized to higher orders by using the expansion eq. (3.22) in the running-coupling evolution equation, to generate recursive equations of the form of eq. (3.23), which determine each $G_{i}$ in terms of the lower-order terms. Note that eq. (3.23) is obtained by neglecting higher order corrections which correspond to commutators of $\widehat{\alpha}_{s}$ and $\chi_{0}$. This terms are nonsingular in the asymptotic limit, but will have to be included explicitly into the kernel when going to yet higher orders. It is clear from eq. (3.23) that these subleading corrections remain perturbative, in the sense that no further singular contributions to $\chi$ are generated beyond those which appear at the leading level. However, the coefficients of these singularities will receive subleading corrections which can be determined by explicit solution of eq. (3.23) and its higher order generalizations.

\section{Conclusions}

In this paper, we have shown how to introduce the running of the coupling to all orders in a BFKL-like $x$-evolution equation, in a way which is consistent with standard renormalization-group evolution. We have thus been able to establish three main results.

First, we have proven that the solution to the running coupling BFKL equation also satisfies a renormalization-group equation, with factorized boundary conditions and a scale dependence entirely determined by an anomalous dimension which is related by duality to the BFKL kernel. This generalizes to the case of all-order running coupling the duality previously derived by us in perturbation theory. Second, by using the well-known Airy solution of this running-coupling BFKL equation with a quadratic kernel, we have shown that the resummed splitting function is free of the unphysical oscillations which characterize the small $x$ behaviour of the solutions to the running-coupling BFKL equation, since these get factorized into the initial condition. Finally, we have shown how the Airy solution can be used to construct a general resummation of running-coupling singular terms, thanks to the fact that the quadratic kernel provides an approximation to the full kernel which 
correctly determines the asymptotic small $x$ behaviour. The net effect of the resummation is small in phenomenologically interesting regions, but it removes a perturbative instability which spoiled the asymptotic small $x$ behaviour of the unresummed results.

In summary, even though it may turn out that in the Regge limit the perturbative behaviour is overwhelmed by power-suppressed contributions, or corrections which go beyond perturbation theory, we have shown that this need not necessarily be so: a leadingtwist perturbative approach may provide a consistent description of scaling violations in the asymptotic small $x$ limit.

Acknowledgements: We thank M. Ciafaloni and G. Salam for various discussions. This work was supported in part by EU TMR contract FMRX-CT98-0194 (DG 12 - MIHT). 


\section{References}

[1] H1 Collab., hep-ex/0012053.

[2] ZEUS Collab., hep-ex/0105090.

[3] V.N. Gribov and L.N. Lipatov, Sov. Jour. Nucl. Phys. 15 (1972) 438;

L.N. Lipatov, Sov. Jour. Nucl. Phys. 20 (1975) 95;

G. Altarelli and G. Parisi, Nucl. Phys. B126 (1977) 298;

see also Y.L. Dokshitzer, Sov. Phys. JETP 46 (1977) 691.

[4] G. Curci, W. Furmański and R. Petronzio, Nucl. Phys. B175 (1980) 27;

E.G. Floratos, C. Kounnas and R. Lacaze, Nucl. Phys. B192 (1981) 417.

[5] S.A. Larin, T. van Ritbergen, J.A.M. Vermaseren, Nucl. Phys. B427 (1994) 41;

S.A. Larin et al., Nucl. Phys. B492 (1997) 338.

[6] L.N. Lipatov, Sov. Jour. Nucl. Phys. 23 (1976) 338;

V.S. Fadin, E.A. Kuraev and L.N. Lipatov, Phys. Lett. 60B (1975) 50; Sov. Phys. JETP 44 (1976) 443; 45 (1977) 199;

Y.Y. Balitski and L.N.Lipatov, Sov. Jour. Nucl. Phys. 28 (1978) 822.

[7] S. Catani and F. Hautmann, Phys. Lett. B315 (1993) 157; Nucl. Phys. B427 (1994) 475.

[8] V.S. Fadin and L.N. Lipatov, Phys. Lett. B429 (1998) 127;

V.S. Fadin et al, Phys. Lett. B359 (1995) 181; Phys. Lett. B387 (1996) 593;

Nucl. Phys. B406 (1993) 259; Phys. Rev. D50 (1994) 5893; Phys. Lett. B389 (1996)

737; Nucl. Phys. B477 (1996) 767; Phys. Lett. B415 (1997) 97; Phys. Lett. B422 (1998) 287.

[9] G. Camici and M. Ciafaloni, Phys. Lett. B412 (1997) 396; Phys. Lett. B430 (1998) 349.

[10] V. del Duca, Phys. Rev. D54 (1996) 989;Phys. Rev. D54 (1996) 4474;

V. del Duca and C.R. Schmidt, Phys. Rev. D57 (1998) 4069;

Z. Bern, V. del Duca and C.R. Schmidt, Phys. Lett. B445 (1998) 168.

[11] T. Jaroszewicz, Phys. Lett. B116 (1982) 291.

[12] S. Catani, F. Fiorani and G. Marchesini, Phys. Lett. B336 (1990) 18;

S. Catani et al., Nucl. Phys. B361 (1991) 645.

[13] A. De Rújula et al., Phys. Rev. D10 (1974) 1649.

[14] R. D. Ball and S. Forte, Phys. Lett. B335 (1994) 77; B336 (1994) 77;

Acta Phys. Polon. B26 (1995) 2097.

[15] R. D. Ball and S. Forte, Phys. Lett. B351 (1995) 313;

R.K. Ellis, F. Hautmann and B.R. Webber, Phys. Lett. B348 (1995) 582.

[16] R. D. Ball and S. Forte, hep-ph/9607291;

I. Bojak and M. Ernst, Phys. Lett. B397 (1997) 296;Nucl. Phys. B508 (1997) 731;

J. Blümlein and A. Vogt, Phys. Rev. D58 (1998) 014020. 
[17] R. D. Ball and S. Forte, hep-ph/9805315;

J. Blümlein et al., hep-ph/9806368.

[18] G. Salam, Jour. High Energy Phys. 9807 (1998) 19.

[19] G. Altarelli, R. D. Ball and S. Forte, Nucl. Phys. B575, 313 (2000); see also hepph/0001157;

[20] G. Altarelli, R. D. Ball and S. Forte, Nucl. Phys. B599 (2001) 383; see also hep$\mathrm{ph} / 0104246$.

[21] M. Ciafaloni and D. Colferai, Phys. Lett. B452 (1999) 372;

M. Ciafaloni, G. Salam and D. Colferai, hep-ph/9905566.

[22] D. A. Ross, Phys. Lett. B 431, 161 (1998)

[23] R. D. Ball and S. Forte, Phys. Lett. B465 (1999) 271.

[24] R. D. Ball and S. Forte, Phys. Lett. B405 (1997) 317.

[25] G. Camici and M. Ciafaloni, Nucl. Phys. B496 (1997) 305.

[26] M. Ciafaloni, D. Colferai and G. P. Salam, JHEP 0007 (2000) 054

[27] L.N. Lipatov, Sov. Phys. J.E.T.P. 63 (1986) 5.

[28] J. C. Collins and J. Kwiecinski, Nucl. Phys. B316 (1989) 307.

[29] Y.V. Kovchegov and A.H. Mueller Phys. Lett. B439 (1998) 428.

[30] N. Armesto, J. Bartels and M. A. Braun, Phys. Lett. B442 (1998) 459.

[31] R. S. Thorne, Phys. Lett. B474 (2000) 372.

[32] M. Ciafaloni, M. Taiuti and A. H. Mueller, hep-ph/0107009.

[33] R. D. Ball and S. Forte, Phys. Lett. B359, 362 (1995).

[34] L. P. A. Haakman, O. V. Kancheli and J. H. Koch Nucl. Phys. B518 (1998) 275. 